\title{
Measurements and identifications of extreme ultraviolet spectra of highly-charged Sm and Er
}

\author{
Y.A. Podpaly, J.D. Gillaspy, J. Reader, Yu. Ralchenko \\ National Institute of Standards and Technology, Gaithersburg, MD 20899, USA \\ E-mail: yuri.podpaly@nist.gov
}

\begin{abstract}
.
We report spectroscopic measurements of highly charged samarium and erbium performed at the National Institute of Standards and Technology (NIST) Electron Beam Ion Trap (EBIT). These measurements are in the extreme ultraviolet (EUV) range, and span electron beam energies from $0.98 \mathrm{keV}$ to $3.00 \mathrm{keV}$. We observed 71 lines from $\mathrm{Kr}$-like $\mathrm{Sm}^{26+}$ to Ni-like $\mathrm{Sm}^{34+}$, connecting 83 energy levels, and 64 lines from Rb-like $\mathrm{Er}^{32+}$ to Ni-like $\mathrm{Er}^{40+}$, connecting 78 energy levels. Of these lines, 64 in $\mathrm{Sm}$ and 60 in $\mathrm{Er}$ are new. Line identifications are performed using collisional-radiative modeling of the EBIT plasma. All spectral lines are assigned individual uncertainties, most in the $\sim 0.001 \mathrm{~nm}$ range. Energy levels are derived from the wavelength measurements.
\end{abstract}




\section{Introduction}

Spectroscopy of rare earth elements has recently become a subject of active research due to the possible use of gadolinium and terbium as next generation light sources for extreme ultraviolet (EUV) lithography [1, 2]. There are also few available data about transitions of highly charged ions in the lanthanides, which makes this an important area for additional study.

In the NIST Atomic Spectra Database [3], erbium and samarium transitions are available primarily for Sm I and II and Er I, II, and III. Fewer data are available for more highly ionized ions. Some transitions have been measured on Electron Beam Ion Traps (EBITs) in the EUV and X-ray regimes [4, 5, 6, 7]. Much of the available data have been generated from laser produced plasmas [8, 9, 10, 11, 12, 13, 14, 15, 16]. Highly charged samarium has also been observed in tokamak plasmas [17, 18, 19]. In highly charged erbium, likewise, there are limited available data, including that generated by EBITs [4, 20, 21], laser plasmas [10, 11, 12, 22], and tokamaks [17, 18, 23].

In this paper, we report Er and $\mathrm{Sm} n=4-\mathrm{n}=4$ transitions in the EUV, continuing on our previous studies of Gd [1] and Dy [24]. A full list of identifications, wavelengths, and wavelength uncertainties is generated, and we calculate energy levels with uncertainties for these ions as well. The intent of this research is to expand the number of measured transitions among the rare earth elements near those of interest for EUV light sources and provide a systematic accounting of uncertainties for transitions and energy levels.

\section{Experiment}

This work was performed at the NIST EBIT [25]. Sm spectra were studied at twelve electron beam energies between $0.98 \mathrm{keV}$ and $2.2 \mathrm{keV}$, and Er spectra were studied at twelve beam energies between $1.3 \mathrm{keV}$ and $3.0 \mathrm{keV}$. These energies are sufficient to produce ions between approximately $\mathrm{Rb}$-like and Ni-like ionization stages [3]. Beam currents varied between $15 \mathrm{~mA}$ and $86 \mathrm{~mA}$. Plasma confinement was achieved, as usual, through the electrostatic trapping via the electron beam, two drift tubes (at $500 \mathrm{~V}$ and $220 \mathrm{~V}$ ), and a $2.8 \mathrm{~T}$ axial magnetic field. Er and Sm were injected into the trap by using a multi-cathode Metal Vapor Vacuum Arc (MeVVA) [26]. The trap was emptied and new ions were injected from the MeVVA every 10 seconds. 
Elements used for calibration were introduced by the MeVVA, by the gas injection system (described in [27]), or were present as intrinsic impurities.

Spectra were recorded with a spectrometer designed for use in the EUV [28]. The spectrometer is a flat-field variable-line spacing type grating spectrometer. Data were collected with a 2048 pixel $\times 512$ pixel $(13.5 \mu \mathrm{m}$ x $13.5 \mu \mathrm{m}$ pixel dimensions $)$ liquid-nitrogen-cooled charge coupled device (CCD). Spectra were taken as ten oneminute exposures, and a cosmic ray filtering program was used to automatically remove data that were outside of five Poisson standard deviations of the signal, effectively removing the majority of the cosmic rays and aberrant electronic noise. The spectral range for Sm measurements covered approximately $4 \mathrm{~nm}$ to $20 \mathrm{~nm}$, and for Er approximately $3 \mathrm{~nm}$ to $17 \mathrm{~nm}$.

Calibration of the samarium spectra was accomplished using twelve lines from $\mathrm{Ne}^{4+}$ through $\mathrm{Ne}^{7+}$, one line of $\mathrm{Fe}^{23+}$, one line of $\mathrm{Fe}^{22+}$, and one line of $\mathrm{Ba}^{26+}$. Spectra of neon were taken at $2 \mathrm{keV}$ and $4 \mathrm{keV}$, iron was taken at $4 \mathrm{keV}$, and barium, which is an intrinsic impurity, was taken at $5.8 \mathrm{keV}$. Calibration of the erbium spectra was performed using twelve lines from $\mathrm{Ne}^{4+}$ through $\mathrm{Ne}^{7+}$, four lines from $\mathrm{Xe}^{43+}$ and $\mathrm{Xe}^{42+}$, one line from $\mathrm{Ba}^{45+}$, one line from $\mathrm{O}^{4+}$, and one line from $\mathrm{O}^{5+}$. Spectra of neon were taken at $2 \mathrm{keV}$ and $4 \mathrm{keV}$, xenon and barium at $5.8 \mathrm{keV}$, and oxygen at $1.8 \mathrm{keV}$. All lines were fit with unweighted Gaussian profiles, and uncertainties were generated for each calibration point. Third order calibration polynomials relating wavelength to detector channel number were calculated, and confidence intervals were generated from which calibration uncertainties were derived. By setting the requirement that the calibration polynomial fit had $\chi^{2} \approx n-N$, where $n$ is the number of calibration points and $N$ is the degrees of freedom of the calibration curve [29], the systematic uncertainty was estimated. For samarium, the systematic uncertainty was found to be $0.00055 \mathrm{~nm}$; for erbium, the systematic uncertainty was found to be $0.0010 \mathrm{~nm}$.

Spectra were recorded at a variety of energies; typical results are shown in figures 1 and 2. The intensities of the experimental spectra are given in the analogto-digital units (ADU) of the CCD. Lines were fit with unweighted Gaussians, and statistical, systematic, and calibration confidence interval uncertainties were added in quadrature for each line to generate a total uncertainty. 


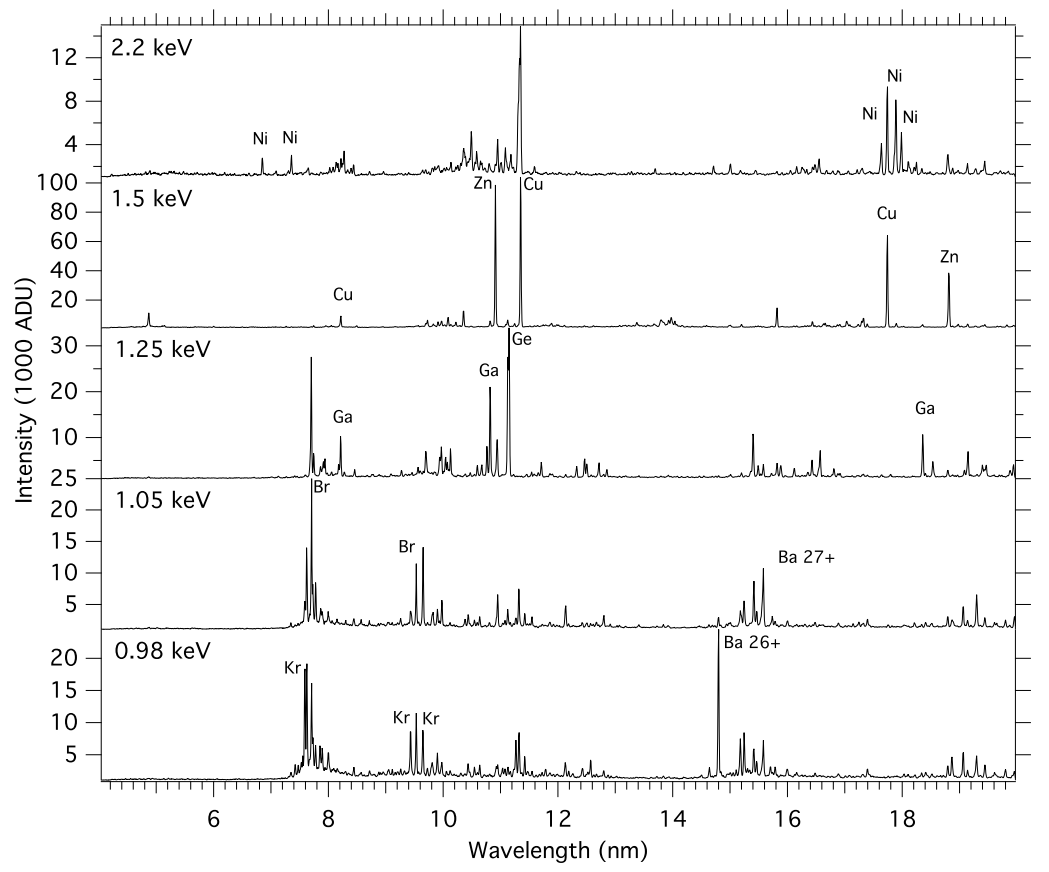

Figure 1. Samarium experimental results at five beam energies. Select lines are labeled by their isoelectronic sequence.

\section{Collisional-Radiative Modeling}

The measured spectra were analyzed with the collisional-radiative (CR) modeling that has been extensively described elsewhere (see, e.g., [30, 31, 32]), and therefore only the most relevant features will be described below. The line intensities for Er and Sm were calculated with the non-Maxwellian CR code NOMAD [33] utilizing atomic data generated with the Flexible Atomic Code (FAC) 34]. The level energies, radiative transition probabilities (allowed and forbidden), and electron-impact cross sections (excitation, deexcitation, ionization, and radiative recombination) were calculated for 8575 and 8570 levels in Sr-like to Ni-like ions of Er and Sm, respectively. The level energies were improved using an extended calculation taking into account all possible excitations within the $n=4$ complex, as described in [31]. The energies of the $3 d^{9} 4 l$ levels in Ni-like ions were taken from a more accurate relativistic many-body perturbation theory (RMBPT) calculation of [35]. The rate of charge exchange between highly-charged ions and neutral atoms in the trap was included 


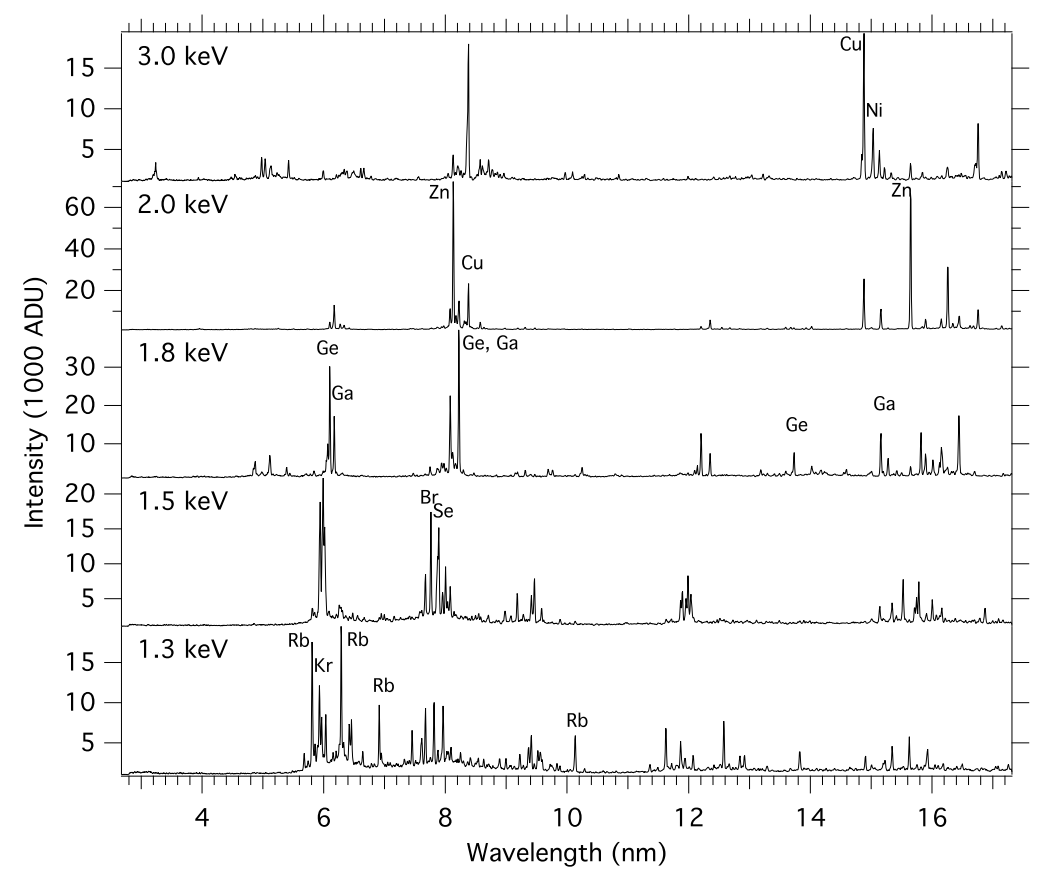

Figure 2. Erbium experimental results at five beam energies. Select lines are labeled by their isoelectronic sequence.

as the only free parameter, aside from a small shift in electron beam energy due to space charge as discussed below. A typical calculation of an EBIT spectrum would include 6-7 ionization stages since ions with ionization potentials larger than the beam energy are very weakly populated. The calculated bound-bound spectra were then Gaussian-broadened and convolved with the calculated efficiency curve of the EUV spectrometer.

Examples of agreement between theoretical and experimental spectra of Er are presented in figures 3 and 4 . The agreement between theory and experiment for the Sm spectra is practically the same. The second order spectra are shown by the shifted dotted lines. Starting with figure 3 , one can see that our CR modeling explains intensities and positions of all strong spectral lines. Note that the theoretical beam energy is lower than the nominal experimental energy; this is due to the space charge effects that are common in EBITs. Although for some lines there is a small shift in wavelength between theory and experiment, a very good match of line intensities allows us to unambiguously identify all prominent lines in the spectrum. For instance, 


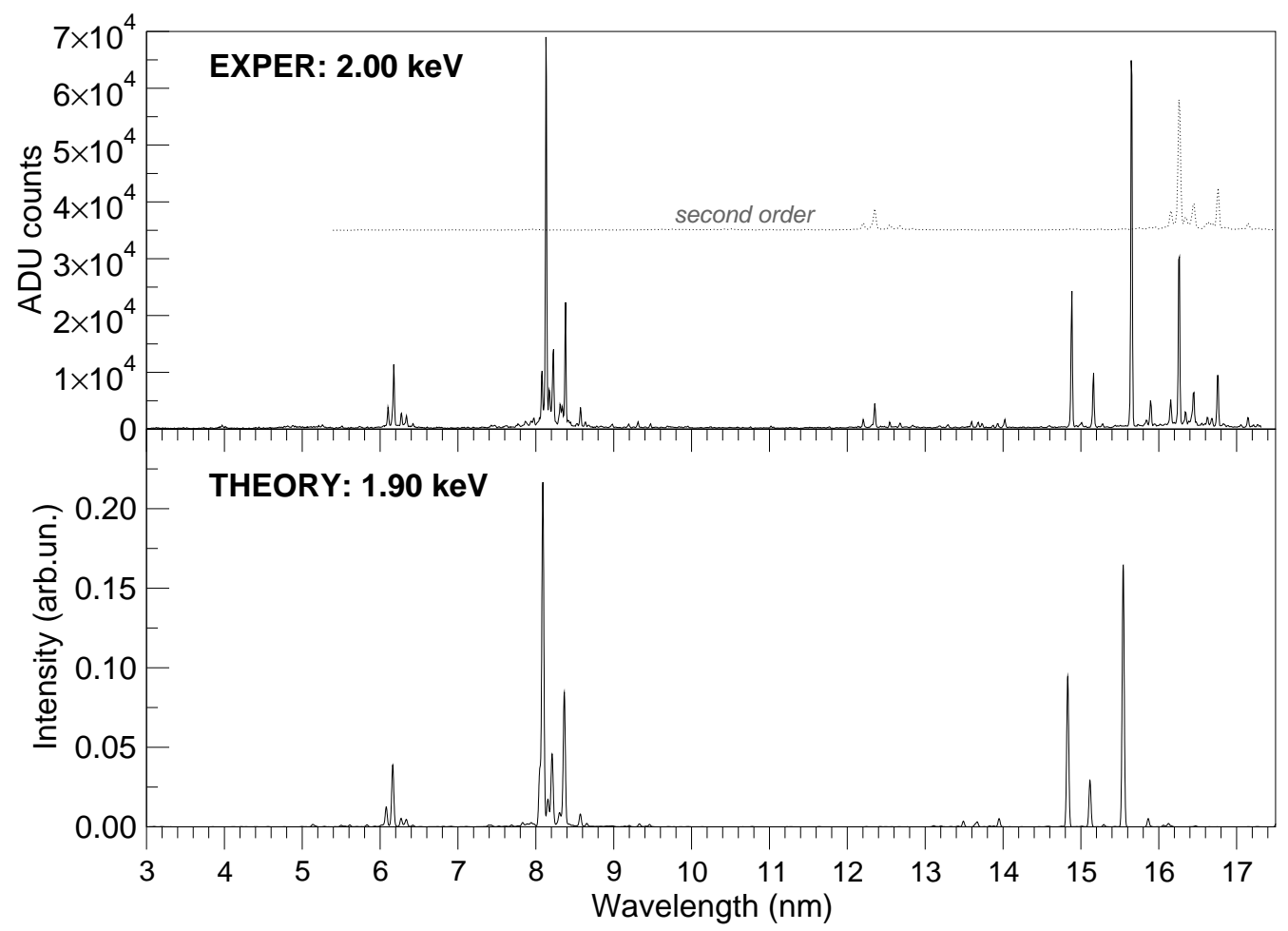

Figure 3. Comparison of the experimental spectrum of erbium with the theoretical spectrum.

the main groups of spectral lines near $8 \mathrm{~nm}$ and $15 \mathrm{~nm}$ are found to be due to the $4 s_{1 / 2}-4 p_{3 / 2}$ and $4 s_{1 / 2}-4 p_{1 / 2}$, respectively, in Cu-, Zn-, and Ga-like ions of Er.

The theoretical spectrum in Fig. 4 is also seen to agree well with the measured spectrum. The contributions from different ions are shown by different colors, while the total theoretical spectrum is marked by a black solid line. For this energy, the calculated ion populations are the following: $[\mathrm{Kr}]:[\mathrm{Br}]:[\mathrm{As}]:[\mathrm{Se}]:[\mathrm{Ge}]=$

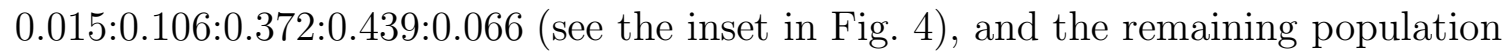
$(<1 \%)$ is in the lower ions. Accordingly, the most prominent lines are due to transitions in As-like (blue) and Ge-like (orange) ions. Although a simple visual comparison allows us to identify practically all experimental lines, additional assistance in identification is provided by comparison of the intensity of the lines at the various beam energies. A similar $E_{\text {beam-dependence of line intensities from a }}$ particular ionization stage is especially helpful in identification of blended lines. 


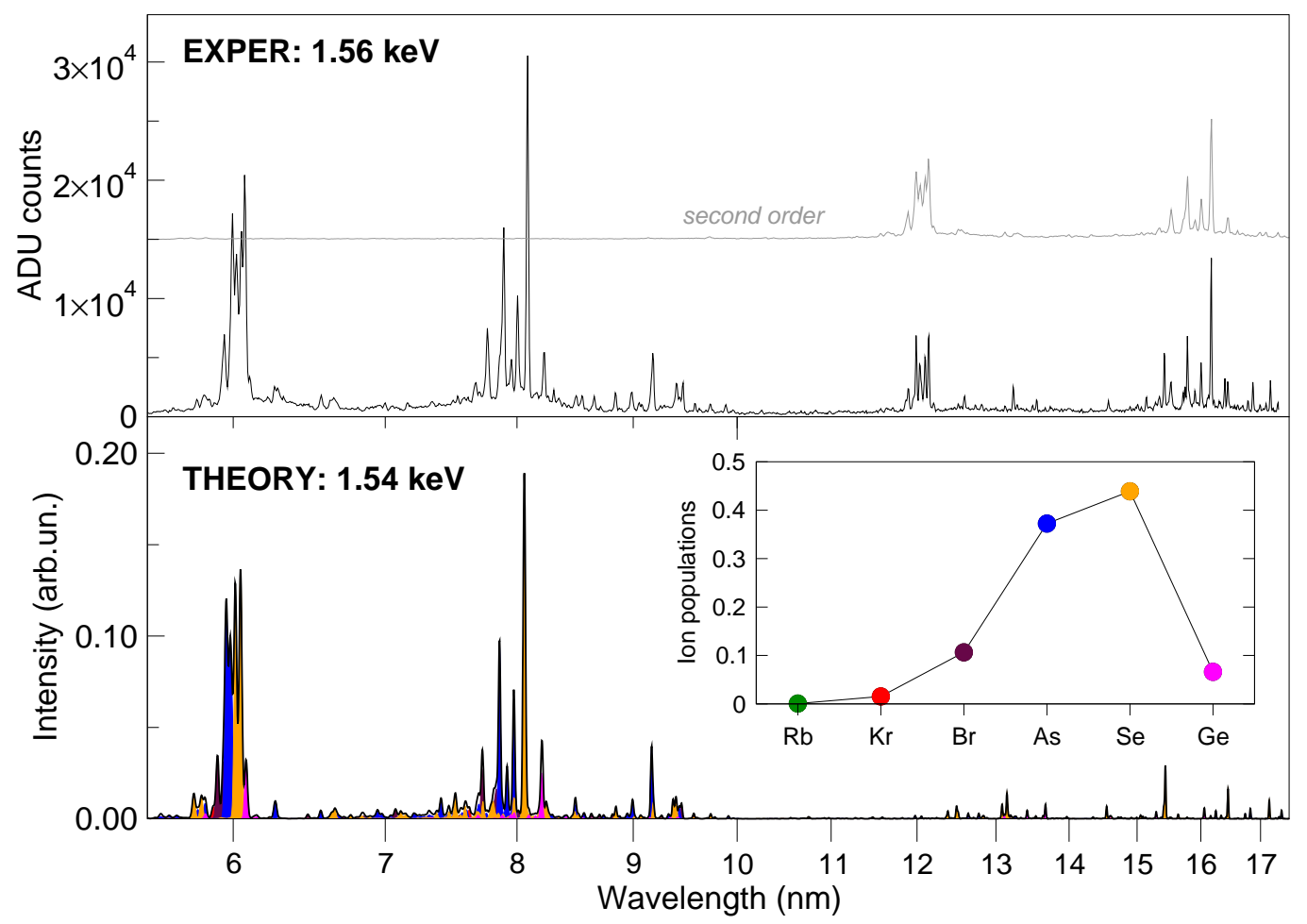

Figure 4. Comparison of the experimental spectrum of erbium with the theoretical spectrum. The inset shows the calculated ionization distribution at $1.54 \mathrm{keV}$. Contributions from different ionization stages are shown in different colors in the theoretical spectrum.

\section{Results}

Results of our measurements are shown in tables 1 and 2. Wavelengths calculated by FAC are presented as well. All uncertainties for wavelengths are reported as one standard deviation. The table also presents previous measurements of several spectral lines. If a line has significant blending with another line the letter ' $b$ ' is appended to the wavelength. For samarium, 71 lines were measured, with 64 of them new lines. For erbium, 64 lines were measured, with 60 of them new lines. In the erbium data, some the of the Br-like lines were measured from their second orders and these wavelengths are marked with '(*2)'.

The level identifications are given in standard notations for relativistic configurations (e.g, $4 p_{+}$for $4 p_{j=l+1 / 2}$ and $4 d_{-}$for $4 d_{j=l-1 / 2}$ ). As is customary for 
the FAC calculations, the electron pairs with total zero momentum are omitted. Since only the largest component of a wavefunction is presented, this may result in non-unique labels for some levels. For instance, levels 7 and 9 in As-like Sm are both shown in table 1 as belonging to the term $4 d_{-}$of configuration $4 p^{2} 4 d$. The calculations show that level 7 is composed of $45.7 \%$ of $4 p^{2} 4 d 4 d_{-}, 45.3 \%$ of $4 s 4 p^{4}$ $\left(4 s_{+},\left(4 p_{+}^{2}\right)_{2}\right)$, and $3.3 \%$ of $4 s 4 p^{4}\left(\left(4 s_{+}, 4 p_{-}\right)_{1},\left(4 p_{+}^{3}\right)_{3 / 2}\right)$, while for level 9 the same relativistic terms have contributions of $49.9 \%, 34.2 \%$ and $5.3 \%$, respectively. Such strong mixing is not uncommon in highly-charged N-shell ions.

The energy levels for samarium and erbium are reported in tables 3 and 4 . The conversion factor from $\mathrm{eV}$ to $\mathrm{cm}^{-1}$ was $1 \mathrm{eV}=8065.544219(18) \mathrm{cm}^{-1}$ [36]. The first energy level, ground level, is taken to have zero energy. In some cases, groups of levels are not connected to the ground level by measured radiative transitions, and therefore the energy of a reference level is taken from FAC or, in the case of Ni-like ions, from Ref. [35]. These levels are marked in our tables with the symbol + followed by a letter. For those levels, the reported results are given as the weighted mean $\left(\propto 1 / \sigma^{2}\right)$ of the possible derivations. Since none of the derived levels have uncertainties less than $20 \mathrm{~cm}^{-1}$, the results are all rounded to $10 \mathrm{~cm}^{-1}$, unless they are from calculations.

For some lines, their identifications were validated by using the Ritz combination principle. For instance, the $8.2227 \mathrm{~nm}$ spectral line in Ga-like Er that corresponds to the transition between the ground state (level 1) and level 6 , is blended by a strong line in the Ge-like ion, and therefore additional confirmation of its wavelength would be helpful. The ground state and the first excited level in this ion are both connected to levels 6 and 7, all four lines having been measured in the present experiment. The energy differences between the two lowest levels calculated from two pairs of measured wavelengths agree within approximately $0.1 \%$, thereby confirming our identifications for all those lines, including the 1-6 transition. Similar analysis was performed for transitions in the Ni-like ions of $\mathrm{Sm}$ and Er.

For the spectral range analyzed here, there exist only a handful of other measurements for Sm and Er. Our wavelengths for Ni-like Sm agree very well with the low-resolution measurements from laser-produced plasmas [9]. The highresolution measurements for $\mathrm{Cu}$-like $\mathrm{Sm}$ and $\mathrm{Er}$ [10, 14] also agree with our results within experimental uncertainties. Similar level of agreement is observed for other measurements in Zn-like ions [11, 12]. This provides additional confidence in our measured wavelengths and identifications of spectral lines from other ions of Sm and 
Er for which no measured lines are known.

For most cases, the FAC wavelengths deviate from the measured values to within a fraction of per cent. The only exception is the transitions in Ni-like Sm that connect level 35 with quantum numbers $3 d^{9} 4 d\left(\left(3 d_{-}^{3}\right)_{3 / 2}, 4 d_{-}\right)_{0}$ with levels 9 and 12 . For this $\mathrm{J}=0$ level the difference between theory and experiment reaches $1.6 \%$ for calculations that include double core excitations from $n=3$ into $n=4$ (value in table 3 ) and $4 \%$ for single 3-4 excitations. The RMBPT results of Ref. [35] do not contain $J=0$ levels and there is no other recent theoretical work addressing the energy of this particular level. We would like to point out that our measured 9-35 and 12-35 wavelengths can provide an interesting test for advanced atomic structure theories.

\section{Conclusions}

We report the results of EUV measurements of highly charged ions of samarium and erbium in an EBIT. One hundred and thirty-five lines are measured with individual uncertainties calculated for each line. From these transitions, a total of 161 energy levels were derived and uncertainties were assigned to each value. Overall, the agreement between theory and experiment is excellent with respect to the intensities of the lines and very good for the wavelengths. Our line identifications agree very well with the already known data for $\mathrm{Ni}-, \mathrm{Cu}-$, and $\mathrm{Zn}-$ like ions.

\section{Acknowledgments}

The authors would like to thank J. Smiga for assistance with calibration of the erbium spectra. This work was funded in part by the Office of Fusion Energy Sciences of the U.S. Department of Energy. Y.P. is supported by a postdoctoral appointment with the National Institute of Standards and Technology National Research Council Research Associateship Program.

\section{References}

[1] Kilbane D, O'Sullivan G, Gillaspy J D, Ralchenko Yu, and Reader J 2012 Phys. Rev. A 86 042503.

[2] Churilov S S, Kildiyarova R R, Ryabtsev A N, and Sadovsky S V 2009 Phys. Scr. 80(4) 045303. 
[3] Kramida A, Ralchenko Yu, Reader J, and NIST ASD Team 2013 NIST Atomic Spectra Database (ver. 5.1), [Online]. Available: http://physics.nist.gov/asd. National Institute of Standards and Technology, Gaithersburg, MD.

[4] Gillaspy J D, Osin D, Ralchenko Yu, Reader J, and Blundell S A 2013 Phys. Rev. A 87062503.

[5] Beiersdorfer P 1993 AIP Conf. Proc. 274(1) 365.

[6] Watanabe H, Currell F J, Fukami T, Kato D, Ohtani S, and Yamada C 2001 Phys. Scr. T92 122 .

[7] Watanabe H, Crosby D, Currell F J, Fukami T, Kato D, Ohtani S, et al. 2001 Phys. Rev. A 63042513.

[8] Louzon D, Henis Z, Levi I, Hurvitz G, Ehrlich Y, Fraenkel M, Maman S, and Mandelbaum P 2009 J. Opt. Soc. Am. B 26(5) 959-964.

[9] Daido H, Ninomiya S, Takagi M, Kato Y, and Koike F 1999 J. Opt. Soc. Am. B 16(2) 296.

[10] Reader J and Luther G 1981Phys. Scr. 24(4) 732.

[11] Reader J and Luther G 1980 Phys. Rev. Lett. 45609.

[12] Acquista N and Reader J 1984 J. Opt. Soc. Am. B 1(4) 649.

[13] Ekberg J O, Feldman U, Seely J F, Brown C M, Reader J, and Acquista N 1987 J. Opt. Soc. Am. B 4(12) 1913.

[14] Doschek G A, Feldman U, Brown C M, Seely J F, Ekberg J O, Behring W E, and Richardson M C 1988 J. Opt. Soc. Am. B 5(2) 243.

[15] Ekberg J O, Feldman U, Hulburt E O, and Reader J 1988 J. Opt. Soc. Am. B 5(6) 1275.

[16] Wyart J F, Fajardo M, Mißualla T, Gauthier J C, Chenais-Popovics C, Klopfel D, et al. 1999 Phys. Scr. T83 35.

[17] Sugar J, Kaufman V, and Rowan W L 1993J. Opt. Soc. Am. B 10(8) 1321.

[18] Sugar J, Kaufman V, and Rowan W L 1993 J. Opt. Soc. Am. B 10(5) 799.

[19] Finkenthal M, Moos H W, Bar-Shalom A, Spector N, Zigler A, and Yarkoni E 1988Phys. Rev. A 38(1) 288.

[20] Elliott S R, Beiersdorfer P, and Nilsen J 1995 Phys. Rev. A 511683.

[21] Elliott S R, Beiersdorfer P, MacGowan B J, and Nilsen J 1995 Phys. Rev. A 522689.

[22] Reader J 1983 J. Opt. Soc. Am. 73(1) 63.

[23] Sugar J, Kaufman V, and Rowan W L 1993 J. Opt. Soc. Am. B 10(11) 1977.

[24] Kilbane D, O’Sullivan G, Podpaly Y A, Gillaspy J D, Reader J, Ralchenko Yu 2014 Eur. Phys J. D. $68(8) 222$

[25] Gillaspy J D, Roberts J R, Brown C M, and Feldman U 1997 Phys. Scr. T71 99.

[26] Holland G E, Boyer C N, Seely J F, Tan J N, Pomeroy J D, and Gillaspy J D 2005 Rev. Sci. Instrum. 76073304.

[27] Fahy K, Sokell E, O'Sullivan G, Aguilar A, Pomeroy J M, Tan J N, et al. 2007 Phys. Rev. A 75032520.

[28] Blagojevic B, Le Bigot E O, Fahy K, Aguilar A, Makonyi K, Takacs E, et al. 2005 Rev. Sci. Instrum. 76(8) 083102.

[29] Hughes I and Hase T 2012 Measurements and their Uncertainties (Oxford University Press)

[30] Ralchenko Yu, Draganić I N, Tan J N, Gillaspy J D, Pomeroy J M, Reader J, et al. 2008 J. Phys. B, 41021003. 
[31] Draganić I N, Ralchenko Yu, Reader J, Gillaspy J D, Tan J N, Pomeroy J M, Brewer S M, and Osin D 2011 J. Phys. B, 44:025001.

[32] Ralchenko Yu, Draganić I N, Osin D, Gillaspy J D, and Reader J 2011 Phys. Rev. A 83032517.

[33] Ralchenko Yu and Maron Y 2001 J. Quant. Spectrosc. Ra. 71(2-6) 609.

[34] Gu M F 2008 Can. J. Phys. 86 675-689.

[35] Safronova U I, Safronova A S, Hamasha S M, and Beiersdorfer P. 2006 Atom. Data. Nucl. Data, 92(1) 47.

[36] Mohr P J, Taylor B N, and Newell D B 2012 Rev. Mod. Phys. 841527 
Table 1: Wavelengths (nm) of highly charged samarium. The numbers in parentheses are the wavelength uncertainties in units of the last significant digit. The numbers in square brackets in column "FAC" are the ordinal numbers for the lower and upper levels. b-blended line.

\begin{tabular}{|c|c|c|c|c|c|c|c|}
\hline \multirow[t]{2}{*}{ Stage } & \multicolumn{2}{|c|}{ Lower Level } & \multicolumn{2}{|c|}{ Upper Level } & \multirow[t]{2}{*}{ Wavelength } & \multirow[t]{2}{*}{ FAC } & \multirow[t]{2}{*}{ Prev. Exp. } \\
\hline & Conf. & State & Conf. & State & & & \\
\hline $34+[\mathrm{Ni}]$ & $3 d^{9} 4 p$ & $\left(\left(3 d_{-}^{3}\right)_{3 / 2}, 4 p_{-}\right)_{1}$ & $3 d^{9} 4 d$ & $\left(\left(3 d_{-}^{3}\right)_{3 / 2}, 4 d_{-}\right)_{0}$ & $6.8494(7)$ & $6.7384[9-35]$ & $6.85(2)^{a}$ \\
\hline $34+[\mathrm{Ni}]$ & $3 d^{9} 4 p$ & $\left(\left(3 d_{+}^{5}\right)_{5 / 2}, 4 p_{+}\right)_{1}$ & $3 d^{9} 4 d$ & $\left(\left(3 d_{-}^{3}\right)_{3 / 2}, 4 d_{-}\right)_{0}$ & $7.3563(8)$ & $7.2344[12-35]$ & $7.36(2)^{a}$ \\
\hline $34+[\mathrm{Ni}]$ & $3 d^{9} 4 p$ & $\left(\left(3 d_{+}\right)_{5 / 2}^{5}, 4 p_{+}\right)_{4}$ & $3 d^{9} 4 d$ & $\left(\left(3 d_{+}^{5}\right)_{5 / 2}, 4 d_{+}\right)_{5}$ & $10.4908(11)$ & $10.4800[10-23]$ & \\
\hline $34+[\mathrm{Ni}]$ & $3 d^{9} 4 s$ & $\left(\left(3 d_{+}^{5}\right)_{5 / 2}, 4 s_{+}\right)_{3}$ & $3 d^{9} 4 p$ & $\left(\left(3 d_{+}^{5}\right)_{5 / 2}, 4 p_{-}\right)_{3}$ & $17.6366(17)$ & $17.5836[2-7]$ & \\
\hline $34+[\mathrm{Ni}]$ & $3 d^{9} 4 s$ & $\left(\left(3 d_{+}^{5}\right)_{5 / 2}, 4 s_{+}\right)_{3}$ & $3 d^{9} 4 p$ & $\left(\left(3 d_{+}^{5}\right)_{5 / 2}, 4 p_{-}\right)_{2}$ & $17.8926(14)$ & $17.8722[2-6]$ & \\
\hline $34+[\mathrm{Ni}]$ & $3 d^{9} 4 s$ & $\left(\left(3 d_{+}^{5}\right)_{5 / 2}, 4 s_{+}\right)_{2}$ & $3 d^{9} 4 p$ & $\left(\left(3 d_{+}^{5}\right)_{5 / 2}, 4 p_{-}\right)_{3}$ & $17.9864(16)$ & $17.9661[3-7]$ & \\
\hline $34+[\mathrm{Ni}]$ & $3 d^{9} 4 s$ & $\left(\left(3 d_{-}^{3}\right)_{3 / 2}, 4 s_{+}\right)_{2}$ & $3 d^{9} 4 p$ & $\left(\left(3 d_{-}^{3}\right)_{3 / 2}, 4 p_{-}\right)_{2}$ & $18.1084(20)$ & $18.0985[5-8]$ & \\
\hline $34+[\mathrm{Ni}]$ & $3 d^{9} 4 s$ & $\left(\left(3 d_{+}^{5}\right)_{5 / 2}, 4 s_{+}\right)_{2}$ & $3 d^{9} 4 p$ & $\left(\left(3 d_{+}^{5}\right)_{5 / 2}, 4 p_{-}\right)_{2}$ & $18.2491(16)$ & $18.2674[3-6]$ & \\
\hline $33+[\mathrm{Cu}]$ & $4 p$ & $4 p_{-}$ & $4 d$ & $4 d_{-}$ & $8.2176(7) \mathrm{b}$ & $8.2193[2-4]$ & $\begin{array}{l}8.2206(15)^{b} \\
8.2155(15)^{c}\end{array}$ \\
\hline $33+[\mathrm{Cu}]$ & $4 d$ & $4 d_{+}$ & $4 f$ & $4 f_{+}$ & $10.2238(7)$ & $10.2183[5-7]$ & $\begin{array}{l}10.2249(15)^{b} \\
10.2223(15)^{c}\end{array}$ \\
\hline $33+[\mathrm{Cu}]$ & $4 p$ & $4 p_{+}$ & $4 d$ & $4 d_{+}$ & $10.3555(7)$ & $10.3588[3-5]$ & $\begin{array}{l}10.3571(15)^{b} \\
10.3551(15)^{c}\end{array}$ \\
\hline $33+[\mathrm{Cu}]$ & $4 s$ & $4 s_{+}$ & $4 p$ & $4 p_{+}$ & $11.3516(7)$ & $11.1969[1-3]$ & $\begin{array}{l}11.3509(15)^{b} \\
11.3504(15)^{c}\end{array}$ \\
\hline $33+[\mathrm{Cu}]$ & $4 s$ & $4 s_{+}$ & $4 p$ & $4 p_{-}$ & $17.7416(13)$ & $17.3523[1-2]$ & $17.7450^{d}$ \\
\hline $32+[\mathrm{Zn}]$ & $4 s 4 d$ & $\left(4 s_{+}, 4 d_{+}\right)_{2}$ & $4 s 4 f$ & $\left(4 s_{+}, 4 f_{+}\right)_{3}$ & $9.9127(7)$ & $9.8855[14-30]$ & \\
\hline $32+[\mathrm{Zn}]$ & $4 s 4 p$ & $\left(4 s_{+}, 4 p_{+}\right)_{1}$ & $4 s 4 d$ & $\left(4 s_{+}, 4 d_{+}\right)_{2}$ & $10.0851(7)$ & $10.0602[5-14]$ & \\
\hline $32+[\mathrm{Zn}]$ & $4 s^{2}$ & $\left(4 s_{+}^{2}\right)_{0}$ & $4 s 4 p$ & $\left(4 s_{+}, 4 p_{+}\right)_{1}$ & $10.9125(6)$ & $10.8361[1-5]$ & $10.911(2)_{\stackrel{N}{N}}^{e}$ \\
\hline
\end{tabular}




$\begin{array}{lll}32+[\mathrm{Zn}] & 4 s 4 p & \left(4 s_{+}, 4 p_{-}\right)_{1} \\ 32+[\mathrm{Zn}] & 4 s^{2} & \left(4 s_{+}^{2}\right)_{0} \\ 31+[\mathrm{Ga}] & 4 p & 4 p_{-} \\ 31+[\mathrm{Ga}] & 4 p & 4 p_{-} \\ 31+[\mathrm{Ga}] & 4 p & 4 p_{+} \\ 31+[\mathrm{Ga}] & 4 p & 4 p_{-} \\ 31+[\mathrm{Ga}] & 4 p & 4 p_{+} \\ 31+[\mathrm{Ga}] & 4 p & 4 p_{-} \\ 31+[\mathrm{Ga}] & 4 p & 4 p_{+} \\ 31+[\mathrm{Ga}] & 4 p & 4 p_{-} \\ 31+[\mathrm{Ga}] & 4 p & 4 p_{+} \\ & & \\ 30+[\mathrm{Ge}] & 4 p^{2} & \left(4 p_{-}^{2}\right)_{0} \\ 30+[\mathrm{Ge}] & 4 p^{2} & \left(4 p_{-}, 4 p_{+}\right)_{2} \\ 30+[\mathrm{Ge}] & 4 p^{2} & \left(4 p_{-}^{2}\right)_{0} \\ 30+[\mathrm{Ge}] & 4 p^{2} & \left(4 p_{-}, 4 p_{+}\right)_{1} \\ 30+[\mathrm{Ge}] & 4 p^{2} & \left(4 p_{-}, 4 p_{+}\right)_{2} \\ 30+[\mathrm{Ge}] & 4 s 4 p^{3} & \left(4 s_{+}, 4 p_{+}\right)_{1} \\ 30+[\mathrm{Ge}] & 4 p^{2} & \left(4 p_{-}, 4 p_{+}\right)_{2} \\ 30+[\mathrm{Ge}] & 4 p^{2} & \left(4 p_{-}, 4 p_{+}\right)_{2} \\ 30+[\mathrm{Ge}] & 4 p^{2} & \left(4 p_{-}, 4 p_{+}\right)_{1} \\ 30+[\mathrm{Ge}] & 4 p^{2} & \left(4 p_{-}^{2}\right)_{0} \\ 30+[\mathrm{Ge}] & 4 p^{2} & \left(4 p_{-}, 4 p_{+}\right)_{2} \\ 30+[\mathrm{Ge}] & 4 p^{2} & \left(4 p_{-}, 4 p_{+}\right)_{2} \\ 30+[\mathrm{Ge}] & 4 p^{2} & \left(4 p_{-}, 4 p_{+}\right)_{2} \\ & & \\ 29+[\mathrm{As}] & 4 p^{3} & 4 p_{+} \\ 29+[\mathrm{Gs}] & 4 p^{3} & 4 p_{+} \\ & & \end{array}$

$4 p^{2}$

$4 p^{2} \quad\left(4 p_{-}, 4 p_{+}\right)_{2}$

$4 s 4 p \quad\left(4 s_{+}, 4 p_{-}\right)_{1}$
$4 d \quad 4 d_{-}$
$4 s 4 p_{2} \quad\left(4 s_{+},\left(4 p_{+}^{2}\right)_{2}\right)_{3 / 2}$
$4 d \quad 4 d_{-}$
$4 s 4 p^{2} \quad\left(4 s_{+},\left(4 p_{+}^{2}\right)_{2}\right)_{5 / 2}$
$4 s 4 p^{2} \quad\left(4 s_{+},\left(4 p_{+}\right)_{2}\right)_{3 / 2}$
$4 s 4 p^{2} \quad\left(\left(4 s_{+}, 4 p_{-}\right)_{1}, 4 p_{+}\right)_{1 / 2}$
$4 s 4 p^{2} \quad\left(\left(4 s_{+}, 4 p_{-}\right)_{1}, 4 p_{+}\right)_{1 / 2}$
$4 s 4 p^{2} \quad 4 s_{+}$

$4 s 4 p^{2} \quad\left(\left(4 s_{+}, 4 p_{-}\right)_{1}, 4 p_{+}\right)_{5 / 2}$

$\begin{array}{ll}4 p 4 d & \left(4 p_{-}, 4 d_{-}\right)_{1} \\ 4 p 4 d & \left(4 p_{+}, 4 d_{-}\right)_{3} \\ 4 s 4 p^{3} & \left(\left(4 s_{+}, 4 p_{-}\right)_{1},\left(4 p_{+}^{2}\right)_{0}\right)_{1} \\ 4 p 4 d & \left(4 p_{-}, 4 d_{-}\right)_{1} \\ 4 p 4 d & \left(4 p_{-}, 4 d_{+}\right)_{3} \\ 4 s 4 p^{2} 4 d & \left(4 s_{+}, 4 d_{+}\right)_{2} \\ 4 s 4 d & \left(4 p_{-}, 4 d_{-}\right)_{2} \\ 4 s 4 p^{3} & \left(\left(4 s_{+}, 4 p_{-}\right)_{1},\left(4 p_{+}^{2}\right)_{0}\right)_{1} \\ 4 p 4 p^{3} & \left(\left(4 s_{+}, 4 p_{-}\right)_{1},\left(4 p_{+}^{2}\right)_{2}\right)_{1} \\ 4 s 4 p^{3} & \left(4 s_{+}, 4 p_{+}\right)_{1} \\ 4 p 4 d & \left(4 p_{-}, 4 d_{-}\right)_{2} \\ 4 s 4 p^{3} & \left(\left(4 s_{+}, 4 p_{-}\right)_{1},\left(4 p_{+}^{2}\right)_{2}\right)_{3} \\ 4 s 4 p^{3} & \left(4 s_{+}, 4 p_{+}\right)_{1} \\ & \\ 4 p^{2} 4 d & \left(\left(4 p_{-}, 4 p_{+}\right)_{2}, 4 d_{-}\right)_{1 / 2} \\ 4 p^{2} 4 d & \left(\left(4 p_{-}, 4 p_{+}\right)_{2}, 4 d_{+}\right)_{5 / 2}\end{array}$

$11.2465(8)$

$18.8153(19)$

$11.2393[3-8]$

$18.658[1-3]$

$7.7461(8) \quad 7.7150[1-11]$

$8.2146(7) \quad 8.1951[1-9]$

$10.1294(7) \quad 10.0619[2-11]$

$10.8203(7) \quad 10.7701[1-7]$

10.9448(20)b 10.8942 [2-9]

$11.1279(7) \quad 11.1055$ [1-6]

$16.8114(11) \quad 16.7187[2-6]$

18.3603(16) $18.3159[1-3]$

$19.1476(22) \quad 19.1158[2-5]$

$\begin{array}{ll}7.7020(7) & 7.6492[1-16] \\ 7.9406(7) & 7.8988[3-23] \\ 8.1809(10) & 8.135[1-13] \\ 9.6994(7) \mathrm{b} & 9.6032[2-16] \\ 9.9701(7) & 9.9429[3-15] \\ 10.0424(7) & 10.0244[7-33] \\ 10.6728(9) & 10.6342[3-14] \\ 10.7648(8) & 10.6784[3-13] \\ 10.9423(7) \mathrm{b} & 10.8984[2-12] \\ 11.1501(7) & 11.1261[1-7] \\ 11.7114(8) & 11.7236[3-10] \\ 12.3270(11) & 12.3630[3-9] \\ 16.5715(9) & 16.5015[3-7] \\ & \\ 7.6812(7) \mathrm{b} & 7.6310[1-24] \\ 7.6812(7) \mathrm{b} & 7.6372[1-23]\end{array}$

$10.9101(10)^{f}$

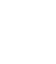




\begin{tabular}{|c|c|c|c|c|c|c|}
\hline $29+[\mathrm{As}]$ & $4 p^{3}$ & $4 p_{+}$ & $4 p^{2} 4 d$ & $\left(\left(4 p_{-}, 4 p_{+}\right)_{2}, 4 d_{-}\right)_{3 / 2}$ & $7.8640(8)$ & $7.8150[1-22]$ \\
\hline $29+[\mathrm{As}]$ & $4 p^{3}$ & $4 p_{+}$ & $4 p^{2} 4 d$ & $\left(\left(4 p_{-}, 4 p_{+}\right)_{1}, 4 d_{+}\right)_{5 / 2}$ & $7.9127(8)$ & $7.8810[1-21]$ \\
\hline $29+[\mathrm{As}]$ & $4 p^{3}$ & $\left(4 p_{-},\left(4 p_{+}^{2}\right)_{2}\right)_{3 / 2}$ & $4 p^{2} 4 d$ & $\left(\left(4 p_{-}, 4 p_{+}\right)_{2}, 4 d_{+}\right)_{5 / 2}$ & $9.0907(8)$ & $8.9946[2-28]$ \\
\hline $29+[\mathrm{As}]$ & $4 p^{3}$ & $4 p_{+}$ & $4 p^{2} 4 d$ & $4 d_{+}$ & $9.9388(8)$ & $9.9104[1-10]$ \\
\hline $29+[\mathrm{As}]$ & $4 p^{3}$ & $4 p_{+}$ & $4 p^{2} 4 d$ & $4 d_{-}$ & $10.5983(7)$ & $10.5592[1-9]$ \\
\hline $29+[\mathrm{As}]$ & $4 p^{3}$ & $4 p_{+}$ & $4 p^{2} 4 d$ & $4 d_{-}$ & $11.5407(7)$ & $11.5409[1-7]$ \\
\hline $29+[\mathrm{As}]$ & $4 p^{3}$ & $4 p_{+}$ & $4 s 4 p^{4}$ & $\left(4 s_{+},\left(4 p_{+}^{2}\right)_{2}\right)_{5 / 2}$ & $12.5081(7)$ & $12.5409[1-6]$ \\
\hline $29+[\mathrm{As}]$ & $4 p^{3}$ & $\left(4 p_{-},\left(4 p_{+}^{2}\right)_{2}\right)_{5 / 2}$ & $4 p^{2} 4 d$ & $4 d_{-}$ & $17.3265(12)$ & $17.3085[3-7]$ \\
\hline $29+[\mathrm{As}]$ & $4 p^{3}$ & $\left(4 p_{-},\left(4 p_{+}^{2}\right)_{0}\right)_{1 / 2}$ & $4 s 4 p^{4}$ & $\left(4 s_{+},\left(4 p_{+}^{2}\right)_{0}\right)_{1 / 2}$ & $17.6760(14)$ & $17.6538[4-8]$ \\
\hline $29+[\mathrm{As}]$ & $4 p^{3}$ & $\left(4 p_{-},\left(4 p_{+}^{2}\right)_{2}\right)_{3 / 2}$ & $4 s 4 p^{4}$ & $\left(4 s_{+},\left(4 p_{+}^{2}\right)_{2}\right)_{5 / 2}$ & $18.4066(17)$ & $18.4182[2-6]$ \\
\hline $29+[\mathrm{As}]$ & $4 p^{3}$ & $\left(4 p_{-},\left(4 p_{+}^{2}\right)_{2}\right)_{5 / 2}$ & $4 s 4 p^{4}$ & $\left(4 s_{+},\left(4 p_{+}^{2}\right)_{2}\right)_{5 / 2}$ & $19.6006(24)$ & $19.6597[3-6]$ \\
\hline $28+[\mathrm{Se}]$ & $4 p^{4}$ & $\left(4 p_{+}^{2}\right)_{2}$ & $4 p^{3} 4 d$ & $\left(\left(4 p_{-},\left(4 p_{+}^{2}\right)_{2}\right)_{3 / 2}, 4 d_{-}\right)_{3}$ & $7.7090(7)$ & $7.6446[1-32]$ \\
\hline $28+[\mathrm{Se}]$ & $4 p^{4}$ & $\left(4 p_{+}^{2}\right)_{2}$ & $4 p^{3} 4 d$ & $\left(\left(4 p_{-},\left(4 p_{+}^{2}\right)_{2}\right)_{5 / 2}, 4 d_{-}\right)_{2}$ & $7.7800(7)$ & $7.7198[1-29]$ \\
\hline $28+[\mathrm{Se}]$ & $4 p^{4}$ & $\left(4 p_{+}^{2}\right)_{2}$ & $4 p^{3} 4 d$ & $\left(4 p_{+}, 4 d_{+}\right)_{3}$ & $9.6502(7) \mathrm{b}$ & $9.6084[1-14]$ \\
\hline $28+[\mathrm{Se}]$ & $4 p^{4}$ & $\left(4 p_{+}^{2}\right)_{0}$ & $4 p^{3} 4 d$ & $\left(4 p_{+}, 4 d_{+}\right)_{1}$ & $9.8209(13)$ & $9.7842[2-15]$ \\
\hline $28+[\mathrm{Se}]$ & $4 p^{4}$ & $\left(4 p_{+}^{2}\right)_{2}$ & $4 p^{3} 4 d$ & $\left(4 p_{+}, 4 d_{+}\right)_{2}$ & $9.9790(8)$ & $9.9450[1-13]$ \\
\hline $28+[\mathrm{Se}]$ & $4 p^{3} 4 d$ & $\left(\left(4 p_{+}^{3}\right)_{3 / 2}, 4 d_{+}\right)_{4}$ & $4 s 4 p^{4} 4 d$ & $\left(\left(4 s_{+},\left(4 p_{+}^{2}\right)_{0}\right)_{1 / 2}, 4 d_{+}\right)_{3}$ & $10.4393(7)$ & $10.4893[12-65$ \\
\hline $28+[\mathrm{Se}]$ & $4 p^{4}$ & $\left(4 p_{+}^{2}\right)_{2}$ & $4 p^{3} 4 d$ & $\left(4 p_{+}, 4 d_{-}\right)_{3}$ & $10.9516(9)$ & $10.9310[1-10]$ \\
\hline $28+[\mathrm{Se}]$ & $4 p^{4}$ & $\left(4 p_{+}^{2}\right)_{2}$ & $4 p^{3} 4 d$ & $\left(4 p_{+}, 4 d_{-}\right)_{1}$ & $11.5467(11)$ & $11.5440[1-7]$ \\
\hline $28+[\mathrm{Se}]$ & $4 p^{4}$ & $\left(4 p_{+}^{2}\right)_{2}$ & $4 s 4 p^{5}$ & $\left(4 s_{+},\left(4 p_{+}\right)_{3 / 2}\right)_{2}$ & $12.1372(8)$ & $12.1546[1-6]$ \\
\hline $27+[\mathrm{Br}]$ & $4 p^{5}$ & $\left(4 p_{+}^{3}\right)_{3 / 2}$ & $4 p^{4} 4 d$ & $\left(\left(4 p_{-},\left(4 p_{+}^{3}\right)_{3 / 2}\right)_{2}, 4 d_{-}\right)_{5 / 2}$ & $7.6242(7)$ & $7.5549[1-27]$ \\
\hline $27+[\mathrm{Br}]$ & $4 p^{5}$ & $\left(4 p_{+}^{3}\right)_{3 / 2}$ & $4 p^{4} 4 d$ & $\left(4 p_{-},\left(4 p_{+}^{3}\right)_{2}, 4 d_{-}\right)_{1 / 2}$ & $7.7321(12)$ & $7.6606[1-25]$ \\
\hline $27+[\mathrm{Br}]$ & $4 p^{5}$ & $\left(4 p_{+}^{3}\right)_{3 / 2}$ & $4 p^{4} 4 d$ & $\left(\left(4 p_{+}^{2}\right)_{2}, 4 d_{+5 / 2}\right)_{5 / 2}$ & $9.5321(7)$ & $9.4986[1-14]$ \\
\hline $27+[\mathrm{Br}]$ & $4 p^{5}$ & $\left(4 p_{+}^{3}\right)_{3 / 2}$ & $4 s$ & $4 s_{+}$ & $9.6479(7) \mathrm{b}$ & $9.6142[1-13]$ \\
\hline $27+[\mathrm{Br}]$ & $4 p^{5}$ & $\left(4 p_{+}^{3}\right)_{3 / 2}$ & $4 p^{4} 4 d$ & $\left(\left(4 p_{+}^{2}\right)_{0}, 4 d_{+5 / 2}\right)_{5 / 2}$ & $9.8126(9)$ & $9.7848[1-12]$ \\
\hline $27+[\mathrm{Br}]$ & $4 p^{5}$ & $\left(4 p_{+}^{3}\right)_{3 / 2}$ & $4 p^{4} 4 d$ & $\left(\left(4 p_{+}^{2}\right)_{2}, 4 d_{+5 / 2}\right)_{1 / 2}$ & $9.9000(7)$ & $9.8682[1-11]$ \\
\hline $26+[\mathrm{Kr}]$ & $4 p^{6}$ & $\left(4 p_{+}^{4}\right)_{0}$ & $4 p^{5} 4 d$ & $\left(4 p_{-}, 4 d_{-}\right)_{1}$ & $7.5901(7)$ & $7.4940[1-13]$ \\
\hline
\end{tabular}




\begin{tabular}{lcccccc}
$26+[\mathrm{Kr}]$ & $4 p^{6}$ & $\left(4 p_{+}^{4}\right)_{0}$ & $4 p^{5} 4 d$ & $\left(\left(4 p_{+}^{3}\right)_{3 / 2}, 4 d_{+}\right)_{1}$ & $9.4330(7)$ & $9.3654[1-9]$ \\
$26+[\mathrm{Kr}]$ & $4 p^{6}$ & $\left(4 p_{+}^{4}\right)_{0}$ & $4 p^{5} 4 d$ & $\left(\left(4 p_{+}^{3}\right)_{3 / 2}, 4 d_{-}\right)_{1}$ & $11.2694(9)$ & $11.2303[1-3]$ \\
\hline${ }^{a}-[9],{ }^{b}-[10],{ }^{c}-[14],{ }^{d}-[19],{ }^{e}-[11],{ }^{f}-[12]$
\end{tabular}


Table 2: Wavelengths (nm) of highly charged erbium. The numbers in parentheses are the wavelength uncertainties in units of the last significant digit. The numbers in square brackets in column "FAC" are the ordinal numbers for the lower and upper levels. b-blended line. (*2)-wavelength derived from second order measurement.

\begin{tabular}{|c|c|c|c|c|c|c|c|}
\hline \multirow[t]{2}{*}{ Stage } & \multicolumn{2}{|c|}{ Lower Level } & \multicolumn{2}{|c|}{ Upper Level } & \multirow[t]{2}{*}{ Wavelength } & \multirow[t]{2}{*}{ FAC } & \multirow[t]{2}{*}{ Prev. Exp. } \\
\hline & Conf. & State & Conf. & State & & & \\
\hline $40+[\mathrm{Ni}]$ & $3 d^{9} 4 p$ & $\left(\left(3 d_{+}^{5}\right)_{5 / 2}, 4 p_{+}\right)_{4}$ & $3 d^{9} 4 d$ & $\left(\left(3 d_{+}^{5}\right)_{5 / 2}, 4 d_{+}\right)_{5}$ & $8.7119(12)$ & $8.7307[10-23]$ & \\
\hline $40+[\mathrm{Ni}]$ & $3 d^{9} 4 s$ & $\left(\left(3 d_{+}^{5}\right)_{5 / 2}, 4 s_{+}\right)_{3}$ & $3 d^{9} 4 p$ & $\left(\left(3 d_{+}^{5}\right)_{5 / 2}, 4 p_{-}\right)_{3}$ & $14.8490(14)$ & $14.8038[2-7]$ & \\
\hline $40+[\mathrm{Ni}]$ & $3 d^{9} 4 s$ & $\left(\left(3 d_{+}^{5}\right)_{5 / 2}, 4 s_{+}\right)_{3}$ & $3 d^{9} 4 p$ & $\left(\left(3 d_{+}^{5}\right)_{5 / 2}, 4 p_{-}\right)_{2}$ & $15.0340(12)$ & $15.0153[2-6]$ & \\
\hline $40+[\mathrm{Ni}]$ & $3 d^{9} 4 s$ & $\left(\left(3 d_{+}^{5}\right)_{5 / 2}, 4 s_{+}\right)_{2}$ & $3 d^{9} 4 p$ & $\left(\left(3 d_{+}^{5}\right)_{5 / 2}, 4 p_{-}\right)_{3}$ & $15.1355(13)$ & $15.1163[3-7]$ & \\
\hline $40+[\mathrm{Ni}]$ & $3 d^{9} 4 s$ & $\left(\left(3 d_{-}^{3}\right)_{3 / 2}, 4 s_{+}\right)_{2}$ & $3 d^{9} 4 p$ & $\left(\left(3 d_{-}^{3}\right)_{3 / 2}, 4 p_{-}\right)_{2}$ & $15.2200(12)$ & $15.2079[5-8]$ & \\
\hline $40+[\mathrm{Ni}]$ & $3 d^{9} 4 s$ & $\left(\left(3 d_{+}^{5}\right)_{5 / 2}, 4 s_{+}\right)_{2}$ & $3 d^{9} 4 p$ & $\left(\left(3 d_{+}^{5}\right)_{5 / 2}, 4 p_{-}\right)_{2}$ & $15.3297(12)$ & $15.3369[3-6]$ & \\
\hline $39+[\mathrm{Cu}]$ & $4 p$ & $4 p_{-}$ & $4 \mathrm{~d}$ & $4 d_{-}$ & $6.3391(14)$ & $6.3379[2-4]$ & $6.3403(15)^{a}$ \\
\hline $39+[\mathrm{Cu}]$ & $4 s$ & $4 s_{+}$ & $4 p$ & $4 p_{+}$ & $8.3796(12)$ & $8.3654[1-3]$ & $8.3813(15)^{a}$ \\
\hline $39+[\mathrm{Cu}]$ & $4 p$ & $4 p_{+}$ & $4 d$ & $4 d_{+}$ & $8.5733(12)$ & $8.5712[3-5]$ & $8.5760(15)^{a}$ \\
\hline $39+[\mathrm{Cu}]$ & $4 s$ & $4 s_{+}$ & $4 p$ & $4 p_{-}$ & $14.8817(13)$ & $14.8305[1-2]$ & \\
\hline $38+[\mathrm{Zn}]$ & $4 s 4 p$ & $\left(4 s_{+}, 4 p_{-}\right)_{1}$ & $4 s 4 d$ & $\left(4 s_{+}, 4 d_{-}\right)_{2}$ & $6.2733(12)$ & $6.2686[3-10]$ & \\
\hline $38+[\mathrm{Zn}]$ & $4 s^{2}$ & $\left(4 s_{+}^{2}\right)_{0}$ & $4 s 4 p$ & $\left(4 s_{+}, 4 p_{+}\right)_{1}$ & $8.1312(12)$ & $8.0900[1-5]$ & $8.131(2)^{b}$ \\
\hline $38+[\mathrm{Zn}]$ & $4 s 4 p$ & $\left(4 s_{+}, 4 p_{+}\right)_{1}$ & $4 s 4 d$ & $\left(4 s_{+}, 4 d_{+}\right)_{2}$ & $8.1732(21)$ & $8.1548[5-14]$ & \\
\hline $38+[\mathrm{Zn}]$ & $4 s^{2}$ & $\left(4 s_{+}^{2}\right)_{0}$ & $4 s 4 p$ & $\left(4 s_{+}, 4 p_{-}\right)_{1}$ & $15.6499(12)$ & $15.5430[1-3]$ & \\
\hline $37+[\mathrm{Ga}]$ & $4 p$ & $4 p_{-}$ & $4 d$ & $4 d_{-}$ & $6.1755(12)$ & $6.1626[1-9]$ & \\
\hline $37+[\mathrm{Ga}]$ & $4 p$ & $4 p_{-}$ & $4 s 4 p^{2}$ & $\left(\left(4 s_{+}, 4 p_{-}\right)_{1}, 4 p_{+}\right)_{1 / 2}$ & $8.0777(14)$ & $8.0497[1-7]$ & \\
\hline $37+[\mathrm{Ga}]$ & $4 p$ & $4 p_{-}$ & $4 s 4 p^{2}$ & $\left(\left(4 s_{+}, 4 p_{-}\right)_{1}, 4 p_{+}\right)_{3 / 2}$ & $8.2227(12) \mathrm{b}$ & $8.2109[1-6]$ & \\
\hline $37+[\mathrm{Ga}]$ & $4 p$ & $4 p_{-}$ & $4 s 4 p^{2}$ & $\left(\left(4 s_{+}, 4 p_{-}\right)_{0}, 4 p_{+}\right)_{3 / 2}$ & $9.3102(12)$ & $9.3281[1-4]$ & \\
\hline $37+[\mathrm{Ga}]$ & $4 p$ & $4 p_{+}$ & $4 s 4 p^{2}$ & $\left(\left(4 s_{+}, 4 p_{-}\right)_{2}, 4 p_{+}\right)_{1 / 2}$ & $13.5942(13)$ & $13.4904[2-7]$ & $\vec{\sigma}$ \\
\hline
\end{tabular}




\begin{tabular}{|c|c|c|c|c|c|c|}
\hline $37+[\mathrm{Ga}]$ & $4 p$ & $4 p_{+}$ & $4 s 4 p^{2}$ & $\left(\left(4 s_{+}, 4 p_{-}\right)_{1}, 4 p_{+}\right)_{3 / 2}$ & $14.0226(13)$ & $13.9493[2-6]$ \\
\hline $37+[\mathrm{Ga}]$ & $4 p$ & $4 p_{-}$ & $4 s 4 p^{2}$ & $4 s_{+}$ & $15.1604(12)$ & $15.1163[1-3]$ \\
\hline $37+[\mathrm{Ga}]$ & $4 p$ & $4 p_{+}$ & $4 s 4 p^{2}$ & $\left(\left(4 s_{+}, 4 p_{-}\right)_{1}, 4 p_{+}\right)_{5 / 2}$ & $15.8957(13)$ & $15.8643[2-5]$ \\
\hline $36+[\mathrm{Ge}]$ & $4 p^{2}$ & $\left(4 p_{-}^{2}\right)_{0}$ & $4 p 4 d$ & $\left(4 p_{-}, 4 d_{-}\right)_{1}$ & $6.1041(12)$ & $6.0786[1-11]$ \\
\hline $36+[\mathrm{Ge}]$ & $4 p^{2}$ & $\left(4 p_{-}^{2}\right)_{0}$ & $4 s 4 p^{3}$ & $\left(4 s_{+}, 4 p_{+}\right)_{1}$ & $8.2214(12) \mathrm{b}$ & $8.2014[1-7]$ \\
\hline $36+[\mathrm{Ge}]$ & $4 p^{2}$ & $\left(4 p_{-}, 4 p_{+}\right)_{2}$ & $4 s 4 p^{3}$ & $\left(4 s_{+}, 4 p_{+}\right)_{1}$ & $13.7334(12)$ & $13.6693[3-7]$ \\
\hline $35+[\mathrm{As}]$ & $4 p^{3}$ & $4 p_{+}$ & $4 p^{2} 4 d$ & $\left(\left(4 p_{-}, 4 p_{+}\right)_{2}, 4 d_{-}\right)_{3 / 2}$ & $6.0497(13)$ & $6.0137[1-21]$ \\
\hline $35+[\mathrm{As}]$ & $4 p^{3}$ & $4 p_{+}$ & $4 p^{2} 4 d$ & $\left(\left(4 p_{-}, 4 p_{+}\right)_{1}, 4 d_{-}\right)_{5 / 2}$ & $6.0727(23)$ & $6.0435[1-20]$ \\
\hline $35+[\mathrm{As}]$ & $4 p^{3}$ & $\left(4 p_{-},\left(4 p_{+}\right)_{0}\right)_{1 / 2}$ & $4 p^{2} 4 d$ & $\left(\left(4 p_{-}, 4 p_{+}\right)_{1}, 4 d_{+}\right)_{7 / 2}$ & $8.8392(13)$ & $8.8433[3-17]$ \\
\hline $35+[\mathrm{As}]$ & $4 p^{3}$ & $\left(4 p_{-},\left(4 p_{+}^{2}\right)_{2}\right)_{3 / 2}$ & $4 s 4 p^{4}$ & $\left(4 s_{+},\left(4 p_{+}^{2}\right)_{2}\right)_{5 / 2}$ & $15.4209(13)$ & $15.4358[2-7]$ \\
\hline $35+[\mathrm{As}]$ & $4 p^{3}$ & $\left(4 p_{-},\left(4 p_{+}^{2}\right)_{2}\right)_{5 / 2}$ & $4 s 4 p^{4}$ & $\left(4 s_{+},\left(4 p_{+}^{2}\right)_{2}\right)_{5 / 2}$ & $16.3966(13)$ & $16.4483[3-7]$ \\
\hline $35+[\mathrm{As}]$ & $4 p^{3}$ & $\left(4 p_{-},\left(4 p_{+}^{2}\right)_{2}\right)_{5 / 2}$ & $4 p^{2} 4 d$ & $4 d_{-}$ & $17.1697(15)$ & $17.1498[3-6]$ \\
\hline $34+[\mathrm{Se}]$ & $4 p^{4}$ & $\left(4 p_{+}^{2}\right)_{2}$ & $4 p^{3} 4 d$ & $\left(\left(4 p_{-},\left(4 p_{+}^{2}\right)_{2}\right)_{3 / 2}, 4 d_{-}\right)_{3}$ & $5.9929(18)$ & $5.9580[1-30]$ \\
\hline $34+[\mathrm{Se}]$ & $4 p^{4}$ & $\left(4 p_{+}^{2}\right)_{2}$ & $4 p^{3} 4 d$ & $\left(\left(4 p_{-},\left(4 p_{+}^{2}\right)_{2}\right)_{5 / 2}, 4 d_{-}\right)_{2}$ & $6.0230(22)$ & $5.9797[1-29]$ \\
\hline $34+[\mathrm{Se}]$ & $4 p^{4}$ & $\left(4 p_{+}^{2}\right)_{2}$ & $4 p^{3} 4 d$ & $\left(4 p_{+}, 4 d_{+}\right)_{3}$ & $7.8933(12)$ & $7.8593[1-14]$ \\
\hline $34+[\mathrm{Se}]$ & $4 p^{4}$ & $\left(4 p_{+}^{2}\right)_{0}$ & $4 p^{3} 4 d$ & $\left(4 p_{+}, 4 d_{+}\right)_{1}$ & $7.9546(12)$ & $7.9199[2-15]$ \\
\hline $34+[\mathrm{Se}]$ & $4 p^{4}$ & $\left(4 p_{+}^{2}\right)_{2}$ & $4 p^{3} 4 d$ & $\left(4 p_{+}, 4 d_{+}\right)_{2}$ & $8.0042(12)$ & $7.9737[1-13]$ \\
\hline $34+[\mathrm{Se}]$ & $4 p^{4}$ & $\left(4 p_{+}^{2}\right)_{2}$ & $4 p^{3} 4 d$ & $\left(4 p_{+}, 4 d_{-}\right)_{3}$ & $9.1787(15)$ & $9.169[1-9]$ \\
\hline $33+[\mathrm{Br}]$ & $4 p^{5}$ & $\left(4 p_{+}^{3}\right)_{3 / 2}$ & $4 p^{4} 4 d$ & $\left(\left(4 p_{-},\left(4 p_{+}^{3}\right)_{3 / 2}\right)_{2}, 4 d_{-}\right)_{5 / 2}$ & $5.9479(6)\left(*^{2}\right)$ & $5.9049[1-26]$ \\
\hline $33+[\mathrm{Br}]$ & $4 p^{5}$ & $\left(4 p_{+}^{3}\right)_{3 / 2}$ & $4 p^{4} 4 d$ & $\left(\left(4 p_{-},\left(4 p_{+}^{3}\right)_{3 / 2}\right)_{2}, 4 d_{-}\right)_{3 / 2}$ & $5.9789(7) \mathrm{b}\left(*^{*}\right)$ & $5.9390[1-25]$ \\
\hline $33+[\mathrm{Br}]$ & $4 p^{5}$ & $\left(4 p_{+}^{3}\right)_{3 / 2}$ & $4 p^{4} 4 d$ & $\left(\left(4 p_{-},\left(4 p_{+}^{3}\right)_{3 / 2}\right)_{2}, 4 d_{-}\right)_{1 / 2}$ & $5.9789(7) \mathrm{b}\left({ }^{*} 2\right)$ & $5.9443[1-24]$ \\
\hline $33+[\mathrm{Br}]$ & $4 p^{5}$ & $\left(4 p_{+}^{3}\right)_{3 / 2}$ & $4 p^{4} 4 d$ & $\left(\left(4 p_{+}^{2}\right)_{2}, 4 d_{+}\right)_{5 / 2}$ & $7.7630(12)$ & $7.7217[1-14]$ \\
\hline $33+[\mathrm{Br}]$ & $4 p^{5}$ & $\left(4 p_{+}^{3}\right)_{3 / 2}$ & $4 s$ & $4 s_{+}$ & $7.8578(10)$ & $7.8181[1-13]$ \\
\hline $33+[\mathrm{Br}]$ & $4 p^{5}$ & $\left(4 p_{+}^{3}\right)_{3 / 2}$ & $4 p^{4} 4 d$ & $\left(\left(4 p_{+}^{2}\right)_{2}, 4 d_{+}\right)_{3 / 2}$ & $7.8717(13)$ & $7.8352[1-12]$ \\
\hline $33+[\mathrm{Br}]$ & $4 p^{5}$ & $\left(4 p_{+}^{3}\right)_{3 / 2}$ & $4 p^{4} 4 d$ & $\left(\left(4 p_{+}^{2}\right)_{2}, 4 d_{+}\right)_{1 / 2}$ & $9.2830(14)$ & $9.2630[1-6]$ \\
\hline $33+[\mathrm{Br}]$ & $4 p^{5}$ & $\left(4 p_{+}^{3}\right)_{3 / 2}$ & $4 p^{4} 4 d$ & $\left(\left(4 p_{+}^{2}\right)_{2}, 4 d_{-}\right)_{5 / 2}$ & $9.4626(47)$ & $9.4519[1-5]$ \\
\hline
\end{tabular}




\begin{tabular}{|c|c|c|c|c|c|c|}
\hline $33+[\mathrm{Br}]$ & $4 p^{5}$ & $\left(4 p_{+}^{3}\right)_{3 / 2}$ & $4 p^{4} 4 d$ & $\left(\left(4 p_{+}^{2}\right)_{2}, 4 d_{-}\right)_{3 / 2}$ & $9.5823(12)$ & $9.5710[1-3]$ \\
\hline $32+[\mathrm{Kr}]$ & $4 p^{6}$ & $\left(4 p_{+}^{4}\right)_{0}$ & $4 p^{5} 4 d$ & $\left(4 p_{-}, 4 d_{-}\right)_{1}$ & $5.9356(13)$ & $5.8792[1-13]$ \\
\hline $32+[\mathrm{Kr}]$ & $4 p^{6}$ & $\left(4 p_{+}^{4}\right)_{0}$ & $4 p^{5} 4 d$ & $\left(\left(4 p_{+}^{3}\right)_{3 / 2}, 4 d_{+}\right)_{1}$ & $7.6746(12)$ & $7.6221[1-9]$ \\
\hline $32+[\mathrm{Kr}]$ & $4 p^{6}$ & $\left(4 p_{+}^{4}\right)_{0}$ & $4 p^{5} 4 d$ & $\left(\left(4 p_{+}^{3}\right)_{3 / 2}, 4 d_{-}\right)_{1}$ & $9.4129(12)$ & $9.3900[1-3]$ \\
\hline $31+[\mathrm{Rb}]$ & $4 d$ & $4 d_{-}$ & $4 p^{5} 4 d^{2}$ & $\left(4 p_{-},\left(4 d_{-}^{2}\right)_{2}\right)_{3 / 2}$ & $5.8139(13)$ & $5.7583[1-45]$ \\
\hline $31+[\mathrm{Rb}]$ & $4 d$ & $4 d_{-}$ & $4 p^{5} 4 d^{2}$ & $\left(4 p_{-},\left(4 d_{-}^{2}\right)_{0}\right)_{1 / 2}$ & $5.9709(18)$ & $5.9212[1-40]$ \\
\hline $31+[\mathrm{Rb}]$ & $4 d$ & $4 d_{-}$ & $4 p^{5} 4 d^{2}$ & $\left(4 p_{-},\left(4 d_{-}^{2}\right)_{2}\right)_{5 / 2}$ & $6.2905(12)$ & $6.2377[1-34]$ \\
\hline $31+[\mathrm{Rb}]$ & $4 d$ & $4 d_{-}$ & $4 f$ & $4 f_{-}$ & $6.9150(11)$ & $6.8765[1-32]$ \\
\hline $31+[\mathrm{Rb}]$ & $4 d$ & $4 d_{-}$ & $4 p^{5} 4 d^{2}$ & $\left(\left(\left(4 p_{+}^{3}\right)_{3 / 2}, 4 d_{-}\right)_{3}, 4 d_{+}\right)_{1 / 2}$ & $7.4543(12)$ & $7.3946[1-28]$ \\
\hline $31+[\mathrm{Rb}]$ & $4 d$ & $4 d_{-}$ & $4 p^{5} 4 d^{2}$ & $\left(\left(\left(4 p_{+}\right)_{3 / 2}, 4 d_{-}\right)_{3}, 4 d_{+}\right)_{3 / 2}$ & $7.8144(12)$ & $7.7673[1-25]$ \\
\hline $31+[\mathrm{Rb}]$ & $4 d$ & $4 d_{-}$ & $4 p^{5} 4 d^{2}$ & $\left(\left(4 p_{+}^{3}\right)_{3 / 2},\left(4 d_{+}^{2}\right)_{4}\right)_{5 / 2}$ & $7.9633(13) \mathrm{b}$ & $7.9128[1-21]$ \\
\hline $31+[\mathrm{Rb}]$ & $4 d$ & $4 d_{-}$ & $4 p^{5} 4 d^{2}$ & $\left(\left(4 p_{+}^{3}\right)_{3 / 2},\left(4 d_{+}^{2}\right)_{2}\right)_{5 / 2}$ & $7.9633(13) \mathrm{b}$ & $7.9255[1-22]$ \\
\hline $31+[\mathrm{Rb}]$ & $4 d$ & $4 d_{-}$ & $4 p^{5} 4 d^{2}$ & $\left(\left(\left(4 p_{+}^{3}\right)_{3 / 2}, 4 d_{-}\right)_{3}, 4 d_{+}\right)_{5 / 2}$ & $8.2514(12)$ & $8.2165[1-17]$ \\
\hline $31+[\mathrm{Rb}]$ & $4 d$ & $4 d_{-}$ & $4 p^{5} 4 d^{2}$ & $\left(\left(\left(4 p_{+}^{3}\right)_{3 / 2}, 4 d_{-}\right)_{1}, 4 d_{+}\right)_{3 / 2}$ & $8.6289(12)$ & $8.6045[1-11]$ \\
\hline $31+[\mathrm{Rb}]$ & $4 d$ & $4 d_{-}$ & $4 p^{5} 4 d^{2}$ & $\left(\left(4 p_{+}^{3}\right)_{3 / 2},\left(4 d_{-}^{2}\right)_{0}\right)_{3 / 2}$ & $8.8905(13)$ & $8.8592[1-8]$ \\
\hline $31+[\mathrm{Rb}]$ & $4 d$ & $4 d_{+}$ & $4 p^{5} 4 d^{2}$ & $\left(\left(\left(4 p_{+}^{3}\right)_{3 / 2}, 4 d_{-}\right)_{2}, 4 d_{+}\right)_{7 / 2}$ & $8.9963(12)$ & $8.9544[2-16]$ \\
\hline $31+[\mathrm{Rb}]$ & $4 d$ & $4 d_{+}$ & $4 p^{5} 4 d^{2}$ & $\left(\left(\left(4 p_{3}^{3}\right)_{3 / 2}, 4 d_{-}\right)_{3}, 4 d_{+}\right)_{7 / 2}$ & $9.2249(12)$ & $9.1879[2-14]$ \\
\hline $31+[\mathrm{Rb}]$ & $4 d$ & $4 d_{-}$ & $4 p^{5} 4 d^{2}$ & $\left(\left(\left(4 p_{+}^{3}\right)_{3 / 2},\left(4 d_{-}^{2}\right)_{2}\right)_{5 / 2}\right.$ & $9.3670(13)$ & $9.3340[1-5]$ \\
\hline $31+[\mathrm{Rb}]$ & $4 d$ & $4 d_{+}$ & $4 p^{5} 4 d^{2}$ & $\left(\left(4 p_{+}^{3}\right)_{3 / 2},\left(4 d_{-}^{2}\right)_{2}\right)_{7 / 2}$ & $10.1342(12)$ & $10.0913[2-6]$ \\
\hline
\end{tabular}


Table 3: Energy levels of highly charged samarium. RMBPT -relativistic many body perturbation theory [35, FAC flexible atomic code [34], $\dagger$-weighted average from two transitions, $\dagger \dagger$-weighted average from three transitions

\begin{tabular}{|c|c|c|c|c|c|c|}
\hline $\begin{array}{l}\text { Stage and } \\
\text { sequence }\end{array}$ & Configuration & State & $\begin{array}{r}\text { Level number } \\
(\text { FAC })\end{array}$ & $\begin{array}{l}\text { Energy } \\
\left(\mathrm{cm}^{-1}\right)\end{array}$ & & $\begin{array}{r}\text { Unc. } \\
\left(\mathrm{cm}^{-1}\right)\end{array}$ \\
\hline $34+[\mathrm{Ni}]$ & $3 d^{10}$ & $\left(3 d_{+}^{6}\right)_{0}$ & 1 & 0 & & 0 \\
\hline $34+[\mathrm{Ni}]$ & $3 d^{9} 4 s$ & $\left(\left(3 d_{+}^{5}\right)_{5 / 2}, 4 s_{+}\right)_{3}$ & 2 & 7522190 & $+\mathrm{x}$ & RMBPT \\
\hline $34+[\mathrm{Ni}]$ & $3 d^{9} 4 s$ & $\left(\left(3 d_{+}^{5}\right)_{5 / 2}, 4 s_{+}\right)_{2}$ & 3 & 7533150 & $+\mathrm{x}$ & $50 \dagger$ \\
\hline $34+[\mathrm{Ni}]$ & $3 d^{9} 4 s$ & $\left(\left(3 d_{-}^{3}\right)_{3 / 2}, 4 s_{+}\right)_{2}$ & 5 & 7752539 & $+\mathrm{y}$ & RMBPT \\
\hline $34+[\mathrm{Ni}]$ & $3 d^{9} 4 p$ & $\left(\left(3 d_{+}^{5}\right)_{5 / 2}, 4 p_{-}\right)_{2}$ & 6 & 8081080 & $+\mathrm{x}$ & 40 \\
\hline $34+[\mathrm{Ni}]$ & $3 d^{9} 4 p$ & $\left(\left(3 d_{+}^{5}\right)_{5 / 2}, 4 p_{-}\right)_{3}$ & 7 & 8089190 & $+\mathrm{x}$ & 50 \\
\hline $34+[\mathrm{Ni}]$ & $3 d^{9} 4 p$ & $\left(\left(3 d_{-}^{3}\right)_{3 / 2}, 4 p_{-}\right)_{2}$ & 8 & 8304770 & $+\mathrm{y}$ & 60 \\
\hline $34+[\mathrm{Ni}]$ & $3 d^{9} 4 p$ & $\left(\left(3 d_{-}^{3}\right)_{3 / 2}, 4 p_{-}\right)_{1}$ & 9 & 8322930 & $+z$ & RMBPT \\
\hline $34+[\mathrm{Ni}]$ & $3 d^{9} 4 p$ & $\left(\left(3 d_{+}^{5}\right)_{5 / 2}, 4 p_{+}\right)_{4}$ & 10 & 8404586 & $+\mathrm{a}$ & FAC \\
\hline $34+[\mathrm{Ni}]$ & $3 d^{9} 4 p$ & $\left(\left(3 d_{+}^{5}\right)_{5 / 2}, 4 p_{+}\right)_{1}$ & 12 & 8423520 & $+\mathrm{z}$ & 200 \\
\hline $34+[\mathrm{Ni}]$ & $3 d^{9} 4 d$ & $\left(\left(3 d_{+}^{5}\right)_{5 / 2}, 4 d_{+}\right)_{5}$ & 23 & 9357800 & $+\mathrm{a}$ & 100 \\
\hline $34+[\mathrm{Ni}]$ & $3 d^{9} 4 d$ & $\left(\left(3 d_{-}^{3}\right)_{3 / 2}, 4 d_{-}\right)_{0}$ & 35 & 9782910 & $+\mathrm{z}$ & 150 \\
\hline $33+[\mathrm{Cu}]$ & $4 s$ & $4 s_{+}$ & 1 & 0 & & 0 \\
\hline $33+[\mathrm{Cu}]$ & $4 p$ & $4 p_{-}$ & 2 & 563650 & & 40 \\
\hline $33+[\mathrm{Cu}]$ & $4 p$ & $4 p_{+}$ & 3 & 880940 & & 50 \\
\hline $33+[\mathrm{Cu}]$ & $4 d$ & $4 d_{-}$ & 4 & 1780550 & & 110 \\
\hline $33+[\mathrm{Cu}]$ & $4 d$ & $4 d_{+}$ & 5 & 1846610 & & 80 \\
\hline $33+[\mathrm{Cu}]$ & $4 f$ & $4 f_{+}$ & 7 & 2824720 & & 110 \\
\hline $32+[\mathrm{Zn}]$ & $4 s^{2}$ & $\left(4 s_{+}^{2}\right)_{0}$ & 1 & 0 & & 0 \\
\hline $32+[\mathrm{Zn}]$ & $4 s 4 p$ & $\left(4 s_{+}, 4 p_{-}\right)_{1}$ & 3 & 531480 & & 50 \\
\hline $32+[\mathrm{Zn}]$ & $4 s 4 p$ & $\left(4 s_{+}, 4 p_{+}\right)_{1}$ & 5 & 916380 & & 50 \\
\hline
\end{tabular}




$\begin{array}{lll}32+[\mathrm{Zn}] & 4 p^{2} & \left(4 p_{-}, 4 p_{-}\right)_{2} \\ 32+[\mathrm{Zn}] & 4 s 4 d & \left(4 s_{+}, 4 d_{+}\right)_{2} \\ 32+[\mathrm{Zn}] & 4 s 4 f & \left(4 s_{+}, 4 f_{+}\right)_{3} \\ & & \\ 31+[\mathrm{Ga}] & 4 p & 4 p_{-} \\ 31+[\mathrm{Ga}] & 4 p & 4 p_{+} \\ 31+[\mathrm{Ga}] & 4 s 4 p^{2} & 4 s_{+} \\ 31+[\mathrm{Ga}] & 4 s 4 p^{2} & \left(\left(4 s_{+}, 4 p_{-}\right)_{1}, 4 p_{+}\right)_{5 / 2} \\ 31+[\mathrm{Ga}] & 4 s 4 p^{2} & \left(\left(4 s_{+}, 4 p_{-}\right)_{1}, 4 p_{+}\right)_{3 / 2} \\ 31+[\mathrm{Ga}] & 4 s 4 p^{2} & \left(\left(4 s_{+}, 4 p_{-}\right)_{1}, 4 p_{+}\right)_{1 / 2} \\ 31+[\mathrm{Ga}] & 4 s 4 p^{2} & \left(4 s_{+},\left(4 p_{+}^{2}\right)_{2}\right)_{3 / 2} \\ 31+[\mathrm{Ga}] & 4 d & 4 d_{-} \\ & & \\ 30+[\mathrm{Ge}] & 4 p^{2} & \left(4 p_{-}^{2}\right)_{0} \\ 30+[\mathrm{Ge}] & 4 p^{2} & \left(4 p_{-}, 4 p_{+}\right)_{1} \\ 30+[\mathrm{Ge}] & 4 p^{2} & \left(4 p_{-}, 4 p_{+}\right)_{2} \\ 30+[\mathrm{Ge}] & 4 s 4 p^{3} & \left(4 s_{+}, 4 p_{+}\right)_{1} \\ 30+[\mathrm{Ge}] & 4 s 4 p^{3} & \left(\left(4 s_{+}, 4 p_{-}\right)_{1},\left(4 p_{+}^{2}\right)_{2}\right)_{3} \\ 30+[\mathrm{Ge}] & 4 p 4 d & \left(4 p_{-}, 4 d_{-}\right)_{2} \\ 30+[\mathrm{Ge}] & 4 p 4 p^{3} & \left(\left(4 s_{+}, 4 p_{-}\right)_{1},\left(4 p_{+}^{2}\right)_{2}\right)_{1} \\ 30+[\mathrm{Ge}] & 4 s 4 p^{3} & \left(\left(4 s_{+}, 4 p_{-}\right)_{1},\left(4 p_{+}^{2}\right)_{0}\right)_{1} \\ 30+[\mathrm{Ge}] & 4 p 4 d & \left(4 p_{-}, 4 d_{+}\right)_{2} \\ 30+[\mathrm{Ge}] & 4 p 4 d & \left(4 p_{-}, 4 d_{+}\right)_{3} \\ 30+[\mathrm{Ge}] & 4 p 4 d & \left(4 p_{-}, 4 d_{-}\right)_{1} \\ 30+[\mathrm{Ge}] & 4 p 4 d & \left(4 p_{+}, 4 d_{-}\right)_{3} \\ 30+[\mathrm{Ge}] & 4 s 4 p^{2} 4 d & \left(4 s_{+}, 4 d_{+}\right)_{2} \\ & & \\ 29+[\mathrm{As}] & 4 p^{3} & \left(4 p_{+}^{3}\right)_{3 / 2} \\ 29+[\mathrm{As}] & 4 p^{3} & \left(4 p_{-},\left(4 p_{+}^{2}\right)_{2}\right)_{3 / 2} \\ 29+[\mathrm{As}] & 4 p^{3} & \left(4 p_{-},\left(4 p_{+}^{2}\right)_{2}\right)_{5} / 2 \\ & & \\ 30 & & \\ 30 & & \end{array}$

$\begin{array}{rrr}8 & 1420650 & 80 \\ 14 & 1907940 & 90 \\ 30 & 2916740 & 110\end{array}$




$\begin{array}{lll}29+[\mathrm{As}] & 4 p^{3} & \left(4 p_{-},\left(4 p_{+}^{2}\right)_{0}\right)_{1 / 2} \\ 29+[\mathrm{As}] & 4 s 4 p^{4} & \left(4 s_{+},\left(4 p_{+}^{2}\right)_{2}\right)_{5 / 2} \\ 29+[\mathrm{As}] & 4 p^{2} 4 d & 4 d_{-} \\ 29+[\mathrm{As}] & 4 s 4 p^{3} & \left(4 s_{+},\left(4 p_{+}^{2}\right)_{0}\right)_{1 / 2} \\ 29+[\mathrm{As}] & 4 p^{2} 4 d & 4 d_{-} \\ 29+[\mathrm{As}] & 4 p^{2} 4 d & 4 d_{+} \\ 29+[\mathrm{As}] & 4 p^{2} 4 d & \left(\left(4 p_{-}, 4 p_{+}\right)_{1}, 4 d_{+}\right)_{5 / 2} \\ 29+[\mathrm{As}] & 4 p^{2} 4 d & \left(\left(4 p_{-}, 4 p_{+}\right)_{2}, 4 d_{-}\right)_{3 / 2} \\ 29+[\mathrm{As}] & 4 p^{2} 4 d & \left(\left(4 p_{-}, 4 p_{+}\right)_{2}, 4 d_{+}\right)_{5 / 2} \\ 29+[\mathrm{As}] & 4 p^{2} 4 d & \left(\left(4 p_{-}, 4 p_{+}\right)_{2}, 4 d_{-}\right)_{1 / 2} \\ 29+[\mathrm{As}] & 4 p^{2} 4 d & \left(\left(4 p_{-},\left(4 p_{+}\right)_{2}\right)_{1} 4 d_{+}\right)_{5 / 2} \\ & & \\ 28+[\mathrm{Se}] & 4 p^{4} & \left(4 p_{+}^{2}\right)_{2} \\ 28+[\mathrm{Se}] & 4 p^{4} & \left(4 p_{+}^{2}\right)_{0} \\ 28+[\mathrm{Se}] & 4 s 4 p^{5} & \left(4 s_{+},\left(4 p_{+}^{3}\right)_{3 / 2}\right)_{2} \\ 28+[\mathrm{Se}] & 4 p^{3} 4 d & \left(4 p_{+}, 4 d_{-}\right)_{1} \\ 28+[\mathrm{Se}] & 4 p^{3} 4 d & \left(4 p_{+}, 4 d_{-}\right)_{3} \\ 28+[\mathrm{Se}] & 4 p^{3} 4 d & \left(4 p_{+}, 4 d_{+}\right)_{4} \\ 28+[\mathrm{Se}] & 4 p^{3} 4 d & \left(4 p_{+}, 4 d_{+}\right)_{2} \\ 28+[\mathrm{Se}] & 4 p^{3} 4 d & \left(4 p_{+}, 4 d_{+}\right)_{3} \\ 28+[\mathrm{Se}] & 4 p^{3} 4 d & \left(4 p_{+}, 4 d_{+}\right)_{1} \\ 28+[\mathrm{Se}] & 4 p^{3} 4 d & \left(\left(4 p_{-},\left(4 p_{+}^{2}\right)_{2}\right)_{5 / 2}, 4 d_{-}\right)_{2} \\ 28+[\mathrm{Se}] & 4 p^{3} 4 d & \left(\left(4 p_{-},\left(4 p_{+}^{2}\right)_{2}\right)_{3 / 2}, 4 d_{-}\right)_{3} \\ 28+[\mathrm{Se}] & 4 s 4 p^{4} 4 d & \left(\left(4 s_{+},\left(4 p_{+}^{2}\right)_{0}\right)_{1 / 2}, 4 d_{+}\right)_{3} \\ & & \\ 27+[\mathrm{Br}] & 4 p^{5} & \left(4 p_{+}^{3}\right)_{3 / 2} \\ 27+[\mathrm{Br}] & 4 p^{4} 4 d & \left(\left(4 p_{+}^{2}\right)_{2}, 4 d_{+}\right)_{1 / 2} \\ 27+[\mathrm{Br}] & 4 p^{4} 4 d & \left(\left(4 p_{+}^{2}\right)_{0}, 4 d_{+}\right)_{5 / 2} \\ 27+[\mathrm{Br}] & 4 s & 4 s_{+} \\ 27+[\mathrm{Br}] & 4 p^{4} 4 d & \left(\left(4 p_{+}^{2}\right)_{2}, 4 d_{+}\right)_{5 / 2} \\ & & \end{array}$

$\begin{array}{rrrrr}4 & 331860 & +\mathrm{x} & \mathrm{FAC} \\ 6 & 799490 & & 50 \\ 7 & 866500 & & 50 \\ 8 & 897600 & +\mathrm{x} & 50 \\ 9 & 943540 & & 60 \\ 10 & 1006160 & & 100 \\ 21 & 1263780 & & 120 \\ 22 & 1271620 & & 120 \\ 23 & 1301880 \mathrm{~b} & & 120 \\ 24 & 1301880 \mathrm{~b} & & 120 \\ 28 & 1356240 & & & 120\end{array}$

\begin{tabular}{|c|c|c|c|}
\hline 1 & 0 & & 0 \\
\hline 2 & 53669 & $+\mathrm{x}$ & FAC \\
\hline 6 & 823910 & & 50 \\
\hline 7 & 866050 & & 80 \\
\hline 10 & 913110 & & 70 \\
\hline 12 & 964703 & $+\mathrm{y}$ & FAC \\
\hline 13 & 1002100 & & 80 \\
\hline 14 & 1036250 & & 70 \\
\hline 15 & 1071900 & $+\mathrm{x}$ & 130 \\
\hline 29 & 1285350 & & 120 \\
\hline 32 & 1297190 & & 130 \\
\hline 65 & 1922620 & $+\mathrm{y}$ & 70 \\
\hline 1 & 0 & & 0 \\
\hline 11 & 1010100 & & 70 \\
\hline 12 & 1019100 & & 100 \\
\hline 13 & 1036490 & & 70 \\
\hline 14 & 1049090 & & 80 \\
\hline
\end{tabular}




$\begin{array}{lll}27+[\mathrm{Br}] & 4 p^{4} 4 d & \left(\left(4 p_{-},\left(4 p_{+}^{3}\right)_{3 / 2}\right)_{2}, 4 d_{-}\right)_{1 / 2} \\ 27+[\mathrm{Br}] & 4 p^{4} 4 d & \left(\left(4 p_{-},\left(4 p_{+}^{3}\right)_{3 / 2}\right)_{2}, 4 d_{-}\right)_{5 / 2} \\ & & \\ 26+[\mathrm{Kr}] & 4 p^{6} & \left(4 p_{+}^{4}\right)_{0} \\ 26+[\mathrm{Kr}] & 4 p^{5} 4 d & \left(\left(4 p_{+}^{3}\right)_{3 / 2}, 4 d_{-}\right)_{1} \\ 26+[\mathrm{Kr}] & 4 p^{5} 4 d & \left(\left(4 p_{+}^{3}\right)_{3 / 2}, 4 d_{+}\right)_{1} \\ 26+[\mathrm{Kr}] & 4 p^{5} 4 d & \left(4 p_{-}, 4 d_{-}\right)_{1}\end{array}$

$25 \quad 1293300$

200

$27 \quad 1311620$

120

$\begin{array}{rrrr}1 & & 0 & 0 \\ 3 & & 887360 & 70 \\ 9 & 1 & 060100 & 80 \\ 13 & 1317500 & 120\end{array}$


Table 4: Energy levels of highly charged erbium. RMBPT -relativistic many-body perturbation theory [35, FAC flexible atomic code [34], $\dagger$-weighted average from two transitions

\begin{tabular}{|c|c|c|c|c|c|c|}
\hline $\begin{array}{l}\text { Stage and } \\
\text { sequence }\end{array}$ & Configuration & State & $\begin{array}{r}\text { Level number } \\
\text { (FAC) }\end{array}$ & $\begin{array}{l}\text { Energy } \\
\left(\mathrm{cm}^{-1}\right)\end{array}$ & & $\begin{array}{r}\text { Unc. } \\
\left(\mathrm{cm}^{-1}\right)\end{array}$ \\
\hline $40+[\mathrm{Ni}]$ & $3 d^{10}$ & $\left(3 d_{+}^{6}\right)_{0}$ & 1 & 0 & & 0 \\
\hline $40+[\mathrm{Ni}]$ & $3 d^{9} 4 s$ & $\left(\left(3 d_{+}^{5}\right)_{5 / 2}, 4 s_{+}\right)_{3}$ & 2 & 9931473 & $+\mathrm{x}$ & RMBPT \\
\hline $40+[\mathrm{Ni}]$ & $3 d^{9} 4 s$ & $\left(\left(3 d_{+}^{5}\right)_{5 / 2}, 4 s_{+}\right)_{2}$ & 3 & 9944260 & $+\mathrm{x}$ & $60 \dagger$ \\
\hline $40+[\mathrm{Ni}]$ & $3 d^{9} 4 s$ & $\left(\left(3 d_{-}^{3}\right)_{3 / 2}, 4 s_{+}\right)_{2}$ & 5 & 10294420 & $+\mathrm{y}$ & RMBPT \\
\hline $40+[\mathrm{Ni}]$ & $3 d^{9} 4 p$ & $\left(\left(3 d_{+}^{5}\right)_{5 / 2}, 4 p_{-}\right)_{2}$ & 6 & 10596630 & $+\mathrm{x}$ & 60 \\
\hline $40+[\mathrm{Ni}]$ & $3 d^{9} 4 p$ & $\left(\left(3 d_{+}^{5}\right)_{5 / 2}, 4 p_{-}\right)_{3}$ & 7 & 10604920 & $+\mathrm{x}$ & 70 \\
\hline $40+[\mathrm{Ni}]$ & $3 d^{9} 4 p$ & $\left(\left(3 d_{-}^{3}\right)_{3 / 2}, 4 p_{-}\right)_{2}$ & 8 & 10951450 & $+\mathrm{y}$ & 50 \\
\hline $40+[\mathrm{Ni}]$ & $3 d^{9} 4 p$ & $\left(\left(3 d_{+}^{5}\right)_{5 / 2}, 4 p_{+}\right)_{4}$ & 10 & 11127857 & $+\mathrm{z}$ & FAC \\
\hline $40+[\mathrm{Ni}]$ & $3 d^{9} 4 d$ & $\left(\left(3 d_{+}^{5}\right)_{5 / 2}, 4 d_{+}\right)_{5}$ & 23 & 12275710 & $+\mathrm{z}$ & 160 \\
\hline $39+[\mathrm{Cu}]$ & $4 s$ & $4 s_{+}$ & 1 & 0 & & 0 \\
\hline $39+[\mathrm{Cu}]$ & $4 p$ & $4 p_{-}$ & 2 & 671970 & & 60 \\
\hline $39+[\mathrm{Cu}]$ & $4 p$ & $4 p_{+}$ & 3 & 1193370 & & 170 \\
\hline $39+[\mathrm{Cu}]$ & $4 d$ & $4 d_{-}$ & 4 & 2249480 & & 360 \\
\hline $39+[\mathrm{Cu}]$ & $4 d$ & $4 d_{+}$ & 5 & 2359780 & & 230 \\
\hline $38+[\mathrm{Zn}]$ & $4 s^{2}$ & $\left(4 s_{+}^{2}\right)_{0}$ & 1 & 0 & & 0 \\
\hline $38+[\mathrm{Zn}]$ & $4 s 4 p$ & $\left(4 s_{+}, 4 p_{-}\right)_{1}$ & 3 & 638980 & & 50 \\
\hline $38+[\mathrm{Zn}]$ & $4 s 4 p$ & $\left(4 s_{+}, 4 p_{+}\right)_{1}$ & 5 & 1229820 & & 180 \\
\hline $38+[\mathrm{Zn}]$ & $4 s 4 d$ & $\left(4 s_{+}, 4 d_{-}\right)_{2}$ & 10 & 2233040 & & 310 \\
\hline $38+[\mathrm{Zn}]$ & $4 s 4 d$ & $\left.4 s_{+}, 4 d_{+}\right)_{2}$ & 14 & 2453320 & & 370 \\
\hline $37+[\mathrm{Ga}]$ & $4 p$ & $4 p_{-}$ & 1 & 0 & & 0 \\
\hline $37+[\mathrm{Ga}]$ & $4 p$ & $4 p_{+}$ & 2 & 502760 & & $140 \dagger$ \\
\hline
\end{tabular}




$\begin{array}{lll}37+[\mathrm{Ga}] & 4 s 4 p^{2} & 4 s_{+} \\ 37+[\mathrm{Ga}] & 4 s 4 p^{2} & \left(\left(4 s_{+}, 4 p_{-}\right)_{0}, 4 p_{+}\right)_{3 / 2} \\ 37+[\mathrm{Ga}] & 4 s 4 p^{2} & \left(\left(4 s_{+}, 4 p_{-}\right)_{1}, 4 p_{+}\right)_{5 / 2} \\ 37+[\mathrm{Ga}] & 4 s 4 p^{2} & \left(\left(4 s_{+}, 4 p_{-}\right)_{1}, 4 p_{+}\right)_{3 / 2} \\ 37+[\mathrm{Ga}] & 4 s 4 p^{2} & \left(\left(4 s_{+}, 4 p_{-}\right)_{1}, 4 p_{+}\right)_{1 / 2} \\ 37+[\mathrm{Ga}] & 4 d & 4 d_{-} \\ & & \\ 36+[\mathrm{Ge}] & 4 p^{2} & \left(4 p_{-}^{2}\right)_{0} \\ 36+[\mathrm{Ge}] & 4 p^{2} & \left(4 p_{-}, 4 p_{+}\right)_{2} \\ 36+[\mathrm{Ge}] & 4 s 4 p^{3} & \left(4 s_{+}, 4 p_{+}\right)_{1} \\ 36+[\mathrm{Ge}] & 4 p 4 d & \left(4 p_{-}, 4 d_{-}\right)_{1} \\ & & \\ 35+[\mathrm{As}] & 4 p^{3} & \left(4 p_{+}^{3}\right)_{3 / 2} \\ 35+[\mathrm{As}] & 4 p^{3} & \left(4 p_{-},\left(4 p_{+}^{2}\right)_{2}\right)_{3 / 2} \\ 35+[\mathrm{As}] & 4 p^{3} & \left(4 p_{-},\left(4 p_{+}^{2}\right)_{2}\right)_{5 / 2} \\ 35+[\mathrm{As}] & 4 p^{2} 4 d & 4 d_{-} \\ 35+[\mathrm{As}] & 4 s 4 p^{4} & \left(4 s_{+},\left(4 p_{+}^{2}\right)_{2}\right)_{5 / 2} \\ 35+[\mathrm{As}] & 4 p^{2} 4 d & \left(\left(4 p_{-}, 4 p_{+}\right)_{1}, 4 d_{+}\right)_{7 / 2} \\ 35+[\mathrm{As}] & 4 p^{2} 4 d & \left(\left(4 p_{-}, 4 p_{+}\right)_{1}, 4 d_{-}\right)_{5 / 2} \\ 35+[\mathrm{As}] & 4 p^{2} 4 d & \left(\left(4 p_{-}, 4 p_{+}\right)_{2}, 4 d_{-}\right)_{3 / 2} \\ & & \\ 34+[\mathrm{Se}] & 4 p^{4} & \left(4 p_{+}^{4}\right)_{2} \\ 34+[\mathrm{Se}] & 4 p^{4} & \left(4 p_{+}^{2}\right)_{0} \\ 34+[\mathrm{Se}] & 4 p^{3} 4 d & \left(4 p_{+}, 4 d_{-}\right)_{3} \\ 34+[\mathrm{Se}] & 4 p^{3} 4 d & \left(4 p_{+}, 4 d_{+}\right)_{2} \\ 34+[\mathrm{Se}] & 4 p^{3} 4 d & \left(4 p_{+}, 4 d_{+}\right)_{3} \\ 34+[\mathrm{Se}] & 4 p^{3} 4 d & \left(4 p_{+}, 4 d_{+}\right)_{1} \\ 34+[\mathrm{Se}] & 4 p^{3} 4 d & \left(\left(4 p_{-},\left(4 p_{+}^{2}\right)_{2}\right)_{5 / 2}, 4 d_{-}\right)_{2} \\ 34+[\mathrm{Se}] & 4 p^{3} 4 d & \left(\left(4 p_{-},\left(4 p_{+}^{2}\right)_{2}\right)_{3 / 2}\right)_{3}\end{array}$

610

1074090

1131460

1216140

1237970

1619300

0

488190

1216340

1638250

50

140

240

170

220

320

190

180

330

\section{2}

3

6

7
17

7
17

17

21

0

440827

$479420+\mathrm{x}$

$1061840+\mathrm{x}$

$1089300+\mathrm{x}$

$1610740 \quad+\mathrm{x}$

1646720

1652980

0

FAC

70

90

50

170

310

370

$\begin{array}{rrrlr}1 & & 0 & & 0 \\ 2 & 63215 & +\mathrm{x} & \mathrm{FAC} \\ 9 & 1089480 & & 180 \\ 13 & 1249340 & & 180 \\ 14 & 1266900 & & 190 \\ 15 & 1320360 & +\mathrm{x} & 190 \\ 29 & 1660300 & & 590 \\ 30 & 1668630 & & 490\end{array}$




$\begin{array}{lll}33+[\mathrm{Br}] & 4 p^{5} & \left(4 p_{+}^{3}\right)_{3 / 2} \\ 33+[\mathrm{Br}] & 4 p^{4} 4 d & \left(\left(4 p_{+}^{2}\right)_{2}, 4 d_{-}\right)_{3 / 2} \\ 33+[\mathrm{Br}] & 4 p^{4} 4 d & \left(\left(4 p_{+}^{2}\right)_{2}, 4 d_{-}\right)_{5 / 2} \\ 33+[\mathrm{Br}] & 4 p^{4} 4 d & \left(\left(4 p_{+}^{2}\right)_{2}, 4 d_{-}\right)_{7 / 2} \\ 33+[\mathrm{Br}] & 4 p^{4} 4 d & \left(\left(4 p_{+}^{2}\right)_{2}, 4 d_{+}\right)_{3 / 2} \\ 33+[\mathrm{Br}] & 4 s & 4 s_{+} \\ 33+[\mathrm{Br}] & 4 p^{4} 4 d & \left(\left(4 p_{+}^{2}\right)_{2}, 4 d_{+}\right)_{5 / 2} \\ 33+[\mathrm{Br}] & 4 p^{4} 4 d & \left(\left(4 p_{-},\left(4 p_{+}^{3}\right)_{3 / 2}\right)_{2}, 4 d_{-}\right)_{1 / 2} \\ 33+[\mathrm{Br}] & 4 p^{4} 4 d & \left(\left(4 p_{-},\left(4 p_{+}^{3}\right)_{3 / 2}\right)_{2}, 4 d_{-}\right)_{3 / 2} \\ 33+[\mathrm{Br}] & 4 p^{4} 4 d & \left(\left(4 p_{-},\left(4 p_{+}^{3}\right)_{3 / 2}\right)_{2}, 4 d_{-}\right)_{5 / 2} \\ & & \\ 32+[\mathrm{Kr}] & 4 p^{6} & \left(4 p_{+}^{4}\right)_{0} \\ 32+[\mathrm{Kr}] & 4 p^{5} 4 d & \left(\left(4 p_{+}^{3}\right)_{3 / 2}, 4 d_{-}\right)_{1} \\ 32+[\mathrm{Kr}] & 4 p^{5} 4 d & \left(\left(4 p_{+}^{3}\right)_{3 / 2}, 4 d_{+}\right)_{1} \\ 32+[\mathrm{Kr}] & 4 p^{5} 4 d & \left(4 p_{-}, 4 d_{-}\right)_{1} \\ & & \\ 31+[\mathrm{Rb}] & 4 d & 4 d_{-} \\ 31+[\mathrm{Rb}] & 4 d & 4 d_{+} \\ 31+[\mathrm{Rb}] & 4 p^{5} 4 d^{2} & \left(\left(4 p_{+}^{3}\right)_{3 / 2},\left(4 d_{-}^{2}\right)_{2}\right)_{5 / 2} \\ 31+[\mathrm{Rb}] & 4 p^{5} 4 d^{2} & \left(\left(4 p_{+}^{3}\right)_{3 / 2},\left(4 d_{-}^{2}\right)_{2}\right)_{7 / 2} \\ 31+[\mathrm{Rb}] & 4 p^{5} 4 d^{2} & \left(\left(4 p_{+}^{3}\right)_{3 / 2},\left(4 d_{-}^{0}\right)_{2}\right)_{3 / 2} \\ 31+[\mathrm{Rb}] & 4 p^{5} 4 d^{2} & \left(\left(\left(4 p_{+}^{3}\right)_{3 / 2}, 4 d_{-}\right)_{1}, 4 d_{+}\right)_{3 / 2} \\ 31+[\mathrm{Rb}] & 4 p^{5} 4 d^{2} & \left(\left(\left(4 p_{+}^{3}\right)_{3 / 2}, 4 d_{-}\right)_{3}, 4 d_{+}\right)_{7 / 2} \\ 31+[\mathrm{Rb}] & 4 p^{5} 4 d^{2} & \left(\left(\left(4 p_{+}^{3}\right)_{3 / 2}, 4 d_{-}\right)_{2}, 4 d_{+}\right)_{7 / 2} \\ 31+[\mathrm{Rb}] & 4 p^{5} 4 d^{2} & \left(\left(\left(4 p_{+}^{3}\right)_{3 / 2}, 4 d_{-}\right)_{3}, 4 d_{+}\right)_{5 / 2} \\ 31+[\mathrm{Rb}] & 4 p^{5} 4 d^{2} & \left(\left(4 p_{+}^{3}\right)_{3 / 2},\left(4 d_{+}^{2}\right)_{4}\right)_{5 / 2} \\ 31+[\mathrm{Rb}] & 4 p^{5} 4 d^{2} & \left(\left(4 p_{+}^{3}\right)_{3 / 2},\left(4 d_{+}^{2}\right)_{4}\right)_{5 / 2} \\ 31+[\mathrm{Rb}] & 4 p^{5} 4 d^{2} & \left(\left(\left(4 p_{+}^{3}\right)_{3 / 2}, 4 d_{-}\right)_{3}, 4 d_{+}\right)_{3 / 2} \\ 31+[\mathrm{Rb}] & 4 p^{5} 4 d^{2} & \left(\left(\left(4 p_{+}^{3}\right)_{3 / 2}, 4 d_{-}\right)_{3}, 4 d_{+}\right)_{1 / 2} \\ 31+[\mathrm{Rb}] & 4 f & 4 f_{-} \\ & & \\ 3 f & & \end{array}$

\begin{tabular}{|c|c|c|c|}
\hline 1 & 0 & & 0 \\
\hline 3 & 1043590 & & 130 \\
\hline 5 & 1056800 & & 530 \\
\hline 6 & 1077240 & & 160 \\
\hline 12 & 1270380 & & 210 \\
\hline 13 & 1272610 & & 160 \\
\hline 14 & 1288160 & & 190 \\
\hline 24 & 1672540 & & 190 \\
\hline 25 & 1672540 & & 190 \\
\hline 26 & 1681280 & & 170 \\
\hline 1 & 0 & & 0 \\
\hline 3 & 1062370 & & 130 \\
\hline 9 & 1303010 & & 200 \\
\hline 13 & 1684740 & & 380 \\
\hline 1 & 0 & & 0 \\
\hline 2 & 95819 & $+\mathrm{x}$ & FAC \\
\hline 5 & 1067570 & & 150 \\
\hline 6 & 1082580 & $+\mathrm{x}$ & 120 \\
\hline 8 & 1124800 & & 170 \\
\hline 11 & 1158890 & & 160 \\
\hline 14 & 1179840 & $+\mathrm{x}$ & 140 \\
\hline 16 & 1207390 & $+\mathrm{x}$ & 150 \\
\hline 17 & 1211910 & & 180 \\
\hline 21 & 1255770 & & 200 \\
\hline 22 & 1255770 & & 200 \\
\hline 25 & 1279690 & & 190 \\
\hline 28 & 1341500 & & 210 \\
\hline & 461 & & \\
\hline
\end{tabular}


$31+[\mathrm{Rb}] \quad 4 p^{5} 4 d^{2}$

$31+[\mathrm{Rb}] \quad 4 p^{5} 4 d^{2}$

$31+[\mathrm{Rb}] \quad 4 p^{5} 4 d^{2}$ $\left(4 p_{-},\left(4 d_{-}^{2}\right)_{2}\right)_{5 / 2}$

$\left(4 p_{-},\left(4 d_{-}^{2}\right)_{0}\right)_{1 / 2}$

$\left(4 p_{-},\left(4 d_{+}^{2}\right)_{2}\right)_{5 / 2}$
$34 \quad 1589690$

$40 \quad 1674780$

$45 \quad 1720010$
300

510

400 